\title{
On the least squares estimation of threshold autoregressive and moving-average models*
}

\author{
Dong $\mathrm{Li}^{\dagger}$, Wai Keung Li And Shiqing Ling
}

This paper considers the least squares estimation and establishes its asymptotic theory for threshold autoregressive and moving-average models. Under some mild conditions, it is shown that the estimator of the threshold is $n$-consistent and after normalization it converges weakly to the smallest minimizer of a compound Poisson process, while the estimators of other coefficients are strongly consistent and asymptotically multivariate normal. This paper also provides a numerical method to tabulate the limiting distribution of the estimated threshold in practice. Simulation studies are carried out to assess the performance of the least squares estimation in finite samples.

AMS 2000 SUBJeCt ClASsifications: Primary 62F12, 62M10; secondary 60G10.

Keywords And Phrases: Asymptotic normality, Compound poisson process, Consistency, Least squares estimation, Threshold ARMA model.

\section{INTRODUCTION}

Since the seminal work of Tong (1978), the threshold model has received considerable attention and is extensively investigated by many researchers. Now it becomes a more or less standard model in nonlinear time series and has been widely used in diverse areas, including biological sciences, econometrics, environmental sciences, finance, hydrology, physics, population dynamics. In comparison with other nonlinear time series models existing in literature, one of the leading reasons behind the success of threshold models is that piecewise linear functions can offer a relatively simple and easy-to-handle approximation to the complex nonlinear dynamics, perhaps more importantly, it can offer a reasonable model-interpretation. Threshold models can capture and be capable of producing many nonlinear phenomena, such as amplitude dependent frequencies, asymmetric limit cycle, chaos, jump resonance, harmonic distortion and so on. A fairly comprehensive survey is available in Tong (1990) and a selective review of the history of threshold models is given by Tong (2011).

*Dedicated to Professor Howell Tong on the occasion of his 65th birthday.

${ }^{\dagger}$ Corresponding author.
Although threshold models have many important applications in practice, there is not a complete theory and methodology as compared with the linear ARMA models. Our knowledge on threshold models is still developing, in particular, for the threshold autoregressive and moving average (TARMA) model.

In the past 30 years or so, the study on the threshold model mainly focuses on threshold autoregressive (TAR) models and there is numerous existing literature related to this topic. For example, on the probabilistic structure of the TAR model, some basic results were given by Chan et al. (1985), Chan and Tong (1985) and Tong (1990). More related results can be found in An and Huang (1996), Brockwell et al. (1992), Chen and Tsay (1991), Ling (1999), Liu and Suskov (1992) and so on. On the other hand, the asymptotic theory of the least squares estimation of the two-regime TAR model was established by Chan (1993) and Chan and Tsay (1998), see also Petruccelli (1986) and Qian (1998). The multi-regime TAR model was considered by Li and Ling (2010). It is worth pointing out that Chan (1993) established the limiting distribution of the estimated threshold, which is the smallest minimizer of a two-sided compound Poisson process, when the autoregressive function is not continuous.

In the development of threshold models, the threshold moving-average (TMA) model, which can be viewed as the superposition of the random impulses through the notion of thresholds, i.e. on-off feedback controllers, has been overshadowed by TAR ones. Earlier works on the TMA model mainly focus on the probabilistic structure, see Brockwell et al. (1992), Liu and Susko (1992) and Ling (1999). Recently, more works enrich the literature on TMA models. Ling et al. (2007) and Li, Ling and Tong (2011) considered the existence, strict stationarity, ergodicity and invertibility of TMA models. Gooijer (1998) proposed the maximum likelihood estimation when the driven impulse is normal. $\mathrm{Li}$, Ling and $\mathrm{Li}$ (2010) developed the least squares estimation and established the asymptotic theory for general TMA models. Ling and Tong (2005) studied a quasi-likelihood ratio test with linear MA model as the null hypothesis against the TMA model as the alternative. This test was extended to the heteroscedastic case by $\mathrm{Li}$ and $\mathrm{Li}$ (2008).

Up to date, however, it seems that TARMA models have not attracted too much attention. There are only a few results available in the literature, all of which mainly study probabilistic properties and give some sufficient conditions 
for the (geometric) ergodicity, strict stationarity and existence of the solution to TARMA models, although they are not easy to verify in practice, see Brockwell et al. (1992), Giovanni (2005), Liu and Susko (1992) and Ling (1999). Recently, Chan and Tong (2010) gave a sufficient condition for the invertibility of nonlinear ARMA models. Li and Li (2011) proposed a quasi-likelihood ratio test for ARMA models against their threshold extensions. To the best of our knowledge, the estimation of TARMA models has not been considered yet, which is one of our motivations in this paper.

In the application, Ghaddar and Tong (1981) applied a TAR(11) model to fit the annual sunspot data of the period 1700-1979, see also Tong (1990, page 420), and Gooijer (1998) used a TMA(17) to fit the quarterly US real GNP data covering the period 1947.I-1982.IV. More real examples can be found in Tong (1990), Tsay (1989) and so on. These examples have a common feature that the order of the model is too high. From a practical point of view, TARMA models should have more advantages over pure TAR or TMA models because these models can provide a parsimonious form just like linear ARMA models. This motivates us to study further TARMA models. As a natural generalization of pure TAR and TMA models, it is necessary to establish a unified theory for estimation, which is our other motivation.

In this paper, we shall study the least squares estimation (LSE) of TARMA models and establish its asymptotic theory. Under some mild conditions, it is shown that the estimator of the threshold is $n$-consistent and after normalization it converges weakly to the smallest minimizer of a two-sided compound Poisson process, while the estimators of other coefficients are strongly consistent and asymptotically multivariate normal. This paper also proposes a numerical method to tabulate the limiting distribution of the estimated threshold in practice. Simulation studies are carried out to assess the performance of the least squares estimation in finite samples.

The remainder of the paper is organized as follows. Section 2 introduces the TARMA model and presents its LSE. Section 3 addresses some assumptions and states main results. Section 4 proposes a numerical approach on simulating the limiting distribution of the estimated threshold. Simulation studies are reported in Section 5 and some concluding remarks are given in Section 6. All proofs of Theorems are left in the Appendix.

Throughout the paper, some symbols are conventional. $C$ is a positive constant, which may be different in different places. $a \vee b \equiv \max \{a, b\}$ for some $a$ and $b$. The summation $\sum_{i=1}^{0} \equiv 0$ and the product $\prod_{i=1}^{0} \equiv 1$. I(.) is an indicator function. $R^{k}$ is the Euclidean space of dimension $k$ and $\|\cdot\|$ denotes the Euclidian norm. $o_{p}(1)\left(O_{p}(1)\right)$ denotes a sequence of random numbers converging to zero (bounded) in probability. $\stackrel{d}{\longrightarrow}$ denotes convergence in distribution and $\Longrightarrow$ denotes weak convergence.

\section{MODEL AND LEAST SQUARES ESTIMATION}

A time series $\left\{y_{t}, t=0, \pm 1, \ldots\right\}$ is said to be a TARMA model with order $\left(p_{1}, p_{2}, q_{1}, q_{2}\right)$, abbreviated to TARMA $\left(p_{1}, p_{2}, q_{1}, q_{2}\right)$, if it satisfies

$y_{t}= \begin{cases}\phi_{10}+\sum_{i=1}^{p_{1}} \phi_{1 i} y_{t-i}+\varepsilon_{t}+\sum_{i=1}^{q_{1}} \psi_{1 i} \varepsilon_{t-i}, & \text { if } y_{t-d} \leq r, \\ \phi_{20}+\sum_{i=1}^{p_{2}} \phi_{2 i} y_{t-i}+\varepsilon_{t}+\sum_{i=1}^{q_{2}} \psi_{2 i} \varepsilon_{t-i}, & \text { if } y_{t-d}>r,\end{cases}$

where $\left\{\varepsilon_{t}\right\}$ is a sequence of independent and identically distributed (i.i.d) random variables, $r \in R, p_{i}$ and $q_{i}$ are known nonnegative integers and $d$ is a positive integer. Here, $r$ is called the threshold parameter and $d$ is the delay variable. When $q_{1}=q_{2}=0$, model (2.1) reduces to a pure TAR model. When $p_{1}=p_{2}=0$, model (2.1) is a pure TMA model. If all $\phi_{1 i}=\phi_{2 i}$ and $\psi_{1 i}=\psi_{2 i}$, then model (2.1) becomes a linear ARMA model.

Without loss of generality, we assume that there exist two finite constants $\underline{r}$ and $\bar{r}$ such that $r \in[\underline{r}, \bar{r}]$ since model (2.1) reduces to a linear ARMA model when $r= \pm \infty$, which is not of our interest here. In addition, $d$ is an unknown parameter to be estimated and its true value is $d_{0}$ with $1 \leq d_{0} \leq D_{0}$, where $D_{0}$ is a known positive integer. Let $\boldsymbol{\theta}_{0}=\left(\boldsymbol{\lambda}_{0}^{\prime}, r_{0}, d_{0}\right)^{\prime}$ be the true value of the parameter $\boldsymbol{\theta}=$ $\left(\boldsymbol{\lambda}^{\prime}, r, d\right)^{\prime} \equiv\left(\boldsymbol{\phi}_{1}^{\prime}, \boldsymbol{\psi}_{1}^{\prime}, \boldsymbol{\phi}_{2}^{\prime}, \boldsymbol{\psi}_{2}^{\prime}, r, d\right)^{\prime}$ with $\boldsymbol{\phi}_{i}=\left(\phi_{i 0}, \ldots, \phi_{i p_{i}}\right)^{\prime}$ and $\boldsymbol{\psi}_{i}=\left(\psi_{i 1}, \ldots, \psi_{i q_{i}}\right)^{\prime}$ for $i=1,2$. The parameter space $\Theta$ is denoted by $\Theta=\Lambda \times[\underline{r}, \bar{r}] \times\left\{1, \ldots, D_{0}\right\}$, where $\Lambda$ is a subset of $R^{p_{1}+p_{2}+q_{1}+q_{2}+2}$.

Assume that $\left\{y_{1}, \ldots, y_{n}\right\}$ is a sample from model (2.1) with sample size $n$. Given the initial value $Y_{0}^{*} \equiv\left\{y_{s}: s \leq 0\right\}$, the sum of square errors function $L_{n}(\boldsymbol{\theta})$ is defined as

$$
L_{n}(\boldsymbol{\theta})=\sum_{t=1}^{n} \varepsilon_{t}^{2}(\boldsymbol{\theta})
$$

where

$$
\begin{aligned}
\varepsilon_{t}(\boldsymbol{\theta})= & y_{t}-\left(\phi_{10}+\sum_{i=1}^{p_{1}} \phi_{1 i} y_{t-i}\right) I\left(y_{t-d} \leq r\right) \\
& -\left(\phi_{20}+\sum_{i=1}^{p_{2}} \phi_{2 i} y_{t-i}\right) I\left(y_{t-d}>r\right) \\
& -\left(\sum_{i=1}^{q_{1}} \psi_{1 i} \varepsilon_{t-i}(\boldsymbol{\theta})\right) I\left(y_{t-d} \leq r\right) \\
& -\left(\sum_{i=1}^{q_{2}} \psi_{2 i} \varepsilon_{t-i}(\boldsymbol{\theta})\right) I\left(y_{t-d}>r\right) .
\end{aligned}
$$

The minimizer $\widehat{\boldsymbol{\theta}}_{n}=\left(\widehat{\boldsymbol{\lambda}}_{n}^{\prime}, \widehat{r}_{n}, \widehat{d}_{n}\right)^{\prime}$ of $L_{n}(\boldsymbol{\theta})$ is called the LSE of $\boldsymbol{\theta}_{0}$, that is,

$$
\widehat{\boldsymbol{\theta}}_{n}=\arg \min _{\boldsymbol{\theta} \in \Theta} L_{n}(\boldsymbol{\theta})
$$


Note that $L_{n}(\boldsymbol{\theta})$ is discontinuous in $r$ and $d$. The way to get $\widehat{\boldsymbol{\theta}}_{n}$ is as follows.

\section{Algorithm A}

Step A.1. For each fixed $r \in R$ and $d \in\left\{1, \ldots, D_{0}\right\}$, we minimize $L_{n}(\boldsymbol{\theta})$ and get its minimizer $\widehat{\boldsymbol{\lambda}}_{n}(r, d)$ and min$\left.\operatorname{imum} L_{n}^{*}(r, d) \equiv L_{n}(\boldsymbol{\theta})\right|_{\boldsymbol{\lambda}=\widehat{\boldsymbol{\lambda}}_{n}(r, d)}$.

Step A.2. Since $L_{n}^{*}(r, d)$ only takes on finite possible values, we can get the minimizer $\left(\widehat{r}_{n}, \widehat{d}_{n}\right)^{\prime}$ of $L_{n}^{*}(r, d)$ by a grid search over the set of order statistics of $\left\{y_{1}, \ldots, y_{n}\right\}$ and $\left\{1, \ldots, D_{0}\right\}$.

Step A.3. Using a plug-in method, one can finally get $\widehat{\boldsymbol{\lambda}}_{n}\left(\widehat{r}_{n}, \widehat{d}_{n}\right)$ and $\widehat{\boldsymbol{\theta}}_{n}$.

Generally, there exist infinitely many $r$ such that $L_{n}(\cdot)$ attains its global minimum. One can choose the smallest of these $r$ 's as an estimator of the threshold. According to the procedure for obtaining $\widehat{\boldsymbol{\theta}}_{n}$, it is not hard to show that $\widehat{\boldsymbol{\theta}}_{n}$ is the LSE of $\boldsymbol{\theta}_{0}$.

In practice, the initial value $Y_{0}^{*}$ is not available and hence we have to replace it by some constants, e.g. $Y_{0}^{*}=x \equiv$ $\left\{x_{1}, \ldots\right\}$. Since $\sup _{\boldsymbol{\theta} \in \Theta}\left\|\mathbf{H}_{t, j}(\boldsymbol{\theta})\right\|=O\left(\rho^{j}\right)$ a.s. by Theorem A.1 in Ling and Tong (2005) under Assumption 3.1, where $\mathbf{H}_{t, j}(\boldsymbol{\theta})$ is defined in (3.1) below, we can show that

$$
\sup _{\boldsymbol{\theta} \in \Theta}\left|\varepsilon_{t}^{2}(\boldsymbol{\theta})-\varepsilon_{t}^{2}(\boldsymbol{\theta})\right|_{Y_{0}^{*}=x} \mid=O\left(\rho^{t}\right) \quad \text { a.s. }
$$

for any given $x$ and some $\rho \in(0,1)$. Thus, the initial value will not affect the asymptotic properties of $\widehat{\boldsymbol{\theta}}_{n}$. For simplicity, in what follows, we assume that $Y_{0}^{*}$ is from model (2.1). In this case, $\varepsilon_{t}\left(\boldsymbol{\theta}_{0}\right)=\varepsilon_{t}$.

\section{MAIN RESULTS}

Let $p=p_{1} \vee p_{2}$ and $q=q_{1} \vee q_{2}$. In what follows, $\Theta$ is assumed to be compact. We first give a sufficient condition for the invertibility of model (2.1). This condition is easy to verify in practice and similar to that of TMA models in Ling and Tong (2005).

Assumption 3.1. $\sum_{j=1}^{q}\left|\psi_{i j}\right|<1$, where $\psi_{i j}=0$ for $j>$ $q_{i}, i=1,2$.

If $\psi_{1 j}=\psi_{2 j}, j=1, \ldots, q$, then the invertible region of model (2.1) is the same as that of the ARMA model when $q=1$, but is smaller than that of ARMA model when $q>1$. This assumption can be relaxed when $q>1$. A weaker condition available can be found in Chan and Tong (2010). Under Assumption 3.1 and the condition that $\left\{y_{t}\right\}$ is strictly stationary with $E\left|y_{t}\right|<\infty$, by Theorem A.2 in Ling and Tong
(2005), the residual $\varepsilon_{t}(\boldsymbol{\theta})$ has the following representation:

$$
\begin{aligned}
\varepsilon_{t}(\boldsymbol{\theta})= & \sum_{j=0}^{\infty}\left[\mathbf{e}^{\prime} \mathbf{H}_{t, j}(\boldsymbol{\theta}) \mathbf{e}\right] \\
& \times\left\{y_{t-j}-\left(\phi_{10}+\sum_{i=1}^{p_{1}} \phi_{1 i} y_{t-i-j}\right) I\left(y_{t-d-j} \leq r\right)\right. \\
& \left.-\left(\phi_{20}+\sum_{i=1}^{p_{2}} \phi_{2 i} y_{t-i-j}\right) I\left(y_{t-d-j}>r\right)\right\},
\end{aligned}
$$

where $\mathbf{e}=(1,0, \ldots, 0)_{q \times 1}^{\prime}$ and

$$
\mathbf{H}_{t, j}(\boldsymbol{\theta})=\prod_{i=1}^{j}\left[\mathbf{\Psi}_{2}+\left(\mathbf{\Psi}_{1}-\mathbf{\Psi}_{2}\right) I\left(y_{t-d-i+1} \leq r\right)\right]
$$

for $j \geq 0$ with the convention $\prod_{i=1}^{0}=\mathbf{I}_{q}$, the identity matrix of size $q$, and

$$
\mathbf{\Psi}_{i}=\left(\begin{array}{ccc}
-\psi_{i 1} & \cdots & -\psi_{i q} \\
& \mathbf{I}_{q-1} & \mathbf{0}
\end{array}\right), \quad i=1,2 .
$$

The following result states the strong consistency of the estimator $\widehat{\boldsymbol{\theta}}_{n}$. The proof is similar to that of Theorem 2.1 in $\mathrm{Li}$, Ling and $\mathrm{Li}$ (2010) and hence it is omitted.

Theorem 3.1. Suppose that (i) Assumption 3.1 holds, (ii) $\left\{y_{t}\right\}$ satisfying (2.1) is strictly stationary and ergodic with $E y_{t}^{2}<\infty$, (iii) $\phi_{10} \neq \phi_{20}$ or $\boldsymbol{\psi}_{10} \neq \boldsymbol{\psi}_{20}$ and (iv) $\varepsilon_{t}$ has a bounded, continuous and strictly positive density on $R$ with $E \varepsilon_{t}=0$ and $E \varepsilon_{t}^{2}<\infty$. Then, $\widehat{\boldsymbol{\theta}}_{n} \rightarrow \boldsymbol{\theta}_{0}$ a.s. as $n \rightarrow \infty$.

The condition (iii) in Theorem 3.1 is required to ensure the identification of the threshold. The strong consistency of $\widehat{\boldsymbol{\theta}}_{n}$ holds, regardless if the autoregressive function is continuous or not. This result is similar to that of TAR models in Chan (1993) and Chan and Tsay (1998) and of TMA models in Li, Ling and Li (2010). From Theorem 3.1, we know that $\widehat{d}_{n}$ equals $d_{0}$ eventually. Thus, without loss of generality, we assume that $d$ is known for the remainder. $d$ is deleted from $\boldsymbol{\theta}$, i.e. $\boldsymbol{\theta}=\left(\boldsymbol{\lambda}^{\prime}, r\right)^{\prime}$ and $\Theta$ is modified, accordingly. Denote $\widehat{\boldsymbol{\lambda}}_{n}(r)=\widehat{\boldsymbol{\lambda}}_{n}\left(r, d_{0}\right)$.

To obtain the convergence rate of $\widehat{r}_{n}$ and the uniformly asymptotic normality of $\widehat{\boldsymbol{\lambda}}_{n} \equiv \widehat{\boldsymbol{\lambda}}_{n}\left(\widehat{r}_{n}\right)$, we need another four assumptions as follows.

Assumption 3.2. $\left\{\varepsilon_{t}\right\}$ is a sequence of i.i.d. random variables with zero mean and $E \varepsilon_{t}^{4}<\infty$. The random variable $\varepsilon_{1}$ has a bounded, continuous and positive density $f_{\varepsilon}$ on $R$.

Assumption 3.3. $\left\{y_{t}\right\}$ is strictly stationary with $E y_{t}^{4}<\infty$.

Let $\mathbf{Z}_{t}=\left(y_{t}, \ldots, y_{t-(p \vee d)+1}, \varepsilon_{t}, \ldots, \varepsilon_{t-q+1}\right)^{\prime}$ with $\left\{\varepsilon_{t}, \ldots, \varepsilon_{k}\right\}=\varnothing$ if $k>t$. Then we can rewrite model (2.1) as a Markovian vectorial representation, see equation (3) in Giovanni (2005) or (2.1)-(2.2) in Liu and Susko (1992), that is, $\left\{\mathbf{Z}_{t}\right\}$ is a Markov chain. Denote its $l$-step transition probability by $\mathcal{P}^{l}(\mathbf{z}, A)$, where $\mathbf{z} \in R^{(p \vee d)+q}$ and $A$ is a Borel set of $R^{(p \vee d)+q}$. 
Assumption 3.4. $\left\{\mathbf{Z}_{t}\right\}$ admits a unique invariant measure $\Pi(\cdot)$ and is $V$-uniformly ergodic with $V(\mathbf{z})=1+\|\mathbf{z}\|$.

Actually, Assumption 3.4 is stronger than geometric ergodicity. For the notion of the $V$-uniform ergodicity, see Meyn and Tweedie (1993, Chapter 16). If the initial value $\mathbf{Z}_{0}$ is from the distribution $\Pi(\cdot)$, then Assumption $3.4 \mathrm{im}-$ plies that $\left\{y_{t}\right\}$ is strictly stationary. For a pure TAR model, it is relatively easy to find a sufficient condition on the coefficients to ensure that Assumption 3.4 holds. See Chan (1989, 1993) and Chan and Tong (1985). While for a pure TMA model, Assumption 3.4 is not necessary since the expression of the strictly stationary and ergodic solution to model (2.1) is available and we can find other weaker conditions to substitute it in studying the convergence rate of $\widehat{r}_{n}$, see Li, Ling and Tong (2011) and Li, Ling and Li (2010). As is well known, it is very hard to verify the $V$-uniform ergodicity for general nonlinear time series models in practice. For TARMA model (2.1), if Assumption 3.2, the conditions $\sum_{j=1}^{p}\left|\phi_{i j}\right|<1$ and $r \neq 0$ hold with $\phi_{10}=\phi_{20}=0$, where $\phi_{i j}=0$ for $j>p_{i}, i=1,2$, then there exists a function

$$
V_{1}\left(\mathbf{Z}_{t}\right)=\max _{0 \leq i \leq(p \vee d)-1}\left\{\rho_{0}^{i}\left|y_{t-i}\right|\right\}+\sum_{j=0}^{q-1} L_{j}\left|\varepsilon_{t-j}\right|+1
$$

such that $E\left[V_{1}\left(\mathbf{Z}_{t}\right) \mid \mathbf{Z}_{t-1}=\mathbf{z}\right] \leq \rho_{0} V_{1}(\mathbf{z})+\left[1+\left(L_{0}+1\right) E\left|\varepsilon_{1}\right|\right]$, which implies that $\left\{\mathbf{Z}_{t}\right\}$ is $V_{1}$-uniformly ergodic, where constants $\rho_{0}$ and $\left\{L_{i}\right\}$ satisfy

$$
\begin{aligned}
\rho_{0} & =\left(\max _{i=1,2} \sum_{j=1}^{p}\left|\phi_{i j}\right|\right)^{1 / p} \in(0,1), \\
\rho_{0} L_{q-1} & \geq b \equiv \max _{i=1,2} \max _{1 \leq j \leq q}\left|\psi_{i j}\right|, \\
\rho_{0} L_{i-1} & \geq L_{i}+b, \quad \text { for } i=1, \ldots, q-1 .
\end{aligned}
$$

It is easy to show that there exist positive constants $c_{1}$ and $c_{2}$ such that $c_{1} V(\mathbf{z}) \leq V_{1}(\mathbf{z}) \leq c_{2} V(\mathbf{z})$. Thus, $\left\{\mathbf{Z}_{t}\right\}$ is also $V$ uniformly ergodic by the definition of $V$-uniform ergodicity. Unfortunately, for other cases, how to verify Assumption 3.4 for TARMA models is still an open problem since the irreducibility and T-continuity of $\left\{\mathbf{Z}_{t}\right\}$ has not been solved. For more details, see Giovanni (2005).

Assumption 3.5. There exist nonrandom vectors $\mathbf{w}=$ $\left(1, w_{1}, \ldots, w_{p}\right)^{\prime} \in R^{p}$ with $w_{d}=r_{0}$ and $\mathbf{a} \in R^{q}$ such that $\left(\phi_{10}-\phi_{20}\right)^{\prime} \mathbf{w}+\left(\boldsymbol{\psi}_{10}-\boldsymbol{\psi}_{20}\right)^{\prime} \mathbf{a} \neq 0$, where $\phi_{i j 0}=0$ for $j>p_{i}$ and $\psi_{i j 0}=0$ for $j>q_{i}, i=1,2$.

In Assumption 3.5, $w_{d}$ may not be a component of $\mathbf{w}$ if $d>p$. In this case, Assumption 3.5 is equivalent to the condition $\left\|\phi_{10}-\phi_{20}\right\|+\left\|\boldsymbol{\psi}_{10}-\boldsymbol{\psi}_{20}\right\|>0$. The latter is necessary and sufficient for the identification of the threshold.

When $p=0$, that is, TARMA model (2.1) reduces to a pure TMA model, Assumption 3.5 is identical to Assumption $2.2 \mathrm{in} \mathrm{Li}$, Ling and $\mathrm{Li}(2010)$ since $\left(\boldsymbol{\psi}_{10}-\boldsymbol{\psi}_{20}\right)^{\prime} \mathbf{a} \neq 0$ for some $\mathbf{a} \in R^{q}$ implies that $\left\|\boldsymbol{\psi}_{10}-\boldsymbol{\psi}_{20}\right\|>0$ and in turn $\widehat{r}_{n}$ has an $n$-convergence rate. When $q=0$, e.g., model (2.1) reduces to a pure TAR model, Assumption 3.5 is the same as Condition 4 in Chan (1993), which implies that the autoregressive function is not continuous over the hyperplane $y_{d}=r_{0}$ so that $\widehat{r}_{n}$ has an $n$-convergence rate. Assumption 3.5 plays a similar role in the convergence rate of $\widehat{r}_{n}$ for TARMA models.

For TAR models, from the results in Chan (1993) and Chan and Tsay (1998), we know that the convergence rate of $\widehat{r}_{n}$ heavily depends on the continuity of the autoregressive function over the hyperplane $y_{d}=r_{0}$. For TARMA models, however, the convergence rate of $\widehat{r}_{n}$ depends not only on the continuity of the autoregressive function, but also on the coefficients in the moving-average part. That is, the continuity of the autoregressive function is not a unique determinant for $n$-convergence rate of $\widehat{r}_{n}$. This point is extremely different from TAR models.

If Assumption 3.5 is violated and the threshold can be identified, then $d \leq p$ and model (2.1) becomes

$$
\begin{aligned}
y_{t}= & \phi_{0}+\sum_{\substack{i=1 \\
i \neq d}}^{p} \phi_{i} y_{t-i}+\phi_{d}^{-}\left(y_{t-d}-r\right)^{-} \\
& +\phi_{d}^{+}\left(y_{t-d}-r\right)^{+}+\varepsilon_{t}+\sum_{i=1}^{q} \psi_{i} \varepsilon_{t-i},
\end{aligned}
$$

where $\phi_{i}, \psi_{j}, \phi_{d}^{-}$and $\phi_{d}^{+}$are the parameters, $(y)^{-}=\min \{y$, $0\}$ and $(y)^{+}=\max \{y, 0\}$. Model (3.3) can be regarded as an extension of the TAR model of Chan and Tsay (1998) when the autoregressive function is continuous, under which $\widehat{r}_{n}$ has a $\sqrt{n}$-convergence rate like other coefficients and is asymptotically normal. We conjecture that similar results hold for model (3.3) under some suitable conditions. Here, we will not pursue this problem.

Theorem 3.2 below shows the convergence rates of $\widehat{r}_{n}$ and the uniformly asymptotic normality of $\widehat{\boldsymbol{\lambda}}_{n}\left(\widehat{r}_{n}\right)$.

Theorem 3.2. If Assumptions 3.1-3.5 hold, then

(i) $n\left(\widehat{r}_{n}-r_{0}\right)=O_{p}(1)$,

(ii) $\sqrt{n} \sup _{\left|r-r_{0}\right| \leq B / n}\left\|\widehat{\boldsymbol{\lambda}}_{n}(r)-\widehat{\boldsymbol{\lambda}}_{n}\left(r_{0}\right)\right\|=o_{p}(1)$

for any fixed constant $B \in(0, \infty)$. Furthermore,

$$
\begin{aligned}
\sqrt{n}\left(\widehat{\boldsymbol{\lambda}}_{n}-\boldsymbol{\lambda}_{0}\right)= & \sqrt{n}\left(\widehat{\boldsymbol{\lambda}}_{n}\left(r_{0}\right)-\boldsymbol{\lambda}_{0}\right)+o_{p}(1) \\
& \stackrel{d}{\longrightarrow} \mathcal{N}\left(0, \sigma^{2} \Sigma^{-1}\right) \quad \text { as } n \rightarrow \infty,
\end{aligned}
$$

where $\sigma^{2}=E \varepsilon_{1}^{2}$ and $\Sigma=E\left[\left(\partial \varepsilon_{t}\left(\boldsymbol{\theta}_{0}\right) / \partial \boldsymbol{\lambda}\right)\left(\partial \varepsilon_{t}\left(\boldsymbol{\theta}_{0}\right) / \partial \boldsymbol{\lambda}^{\prime}\right)\right]$.

Generally, since they are unknown in practice, we can estimate $\sigma^{2}$ and $\Sigma$ consistently by

$\widehat{\sigma}_{n}^{2}=\frac{1}{n} \sum_{t=1}^{n} \varepsilon_{t}^{2}\left(\widehat{\boldsymbol{\theta}}_{n}\right) \quad$ and $\quad \widehat{\Sigma}_{n}=\frac{1}{n} \sum_{t=1}^{n} \frac{\partial \varepsilon_{t}\left(\widehat{\boldsymbol{\theta}}_{n}\right)}{\partial \boldsymbol{\lambda}} \frac{\partial \varepsilon_{t}\left(\widehat{\boldsymbol{\theta}}_{n}\right)}{\partial \boldsymbol{\lambda}^{\prime}}$ 
Using these estimators, one can easily construct approximate confidence regions for the coefficient parameters in TARMA models in terms of the limiting distribution in Theorem 3.2. For example, an approximate $(1-\alpha)$ confidence region for the coefficient vector $\boldsymbol{\lambda}$ is obtained as

$$
\left\{\boldsymbol{\lambda}:\left(\widehat{\boldsymbol{\lambda}}_{n}-\boldsymbol{\lambda}\right)^{\prime} \widehat{\Sigma}_{n}\left(\widehat{\boldsymbol{\lambda}}_{n}-\boldsymbol{\lambda}\right) \leq \widehat{\sigma}_{n}^{2} \chi_{p_{1}+p_{2}+q_{1}+q_{2}+2}^{2}(1-\alpha) / n\right\},
$$

where $\chi_{k}^{2}(1-\alpha)$ is the $100 \alpha$ th percentile of the $\chi^{2}$-distribution with $k$ degrees of freedom.

To study the limiting distribution of $\widehat{r}_{n}$, we need to consider the following profile sum of square errors function:

$\tilde{L}_{n}(z)=L_{n}\left(\widehat{\boldsymbol{\lambda}}_{n}\left(r_{0}+\frac{z}{n}\right), r_{0}+\frac{z}{n}\right)-L_{n}\left(\widehat{\boldsymbol{\lambda}}_{n}\left(r_{0}\right), r_{0}\right), z \in R$.

It is not hard to show that $\tilde{L}_{n}(z)$ can be approximated in $D(R)$, the space of all cadlag functions on $R$ being equipped with the Skorokhod metric, by

$$
\begin{aligned}
\wp_{n}(z)= & I(z<0) \sum_{t=1}^{n} \zeta_{1 t} I\left(r_{0}+\frac{z}{n}<y_{t-d} \leq r_{0}\right) \\
& +I(z \geq 0) \sum_{t=1}^{n} \zeta_{2 t} I\left(r_{0}<y_{t-d} \leq r_{0}+\frac{z}{n}\right),
\end{aligned}
$$

where

$$
\begin{aligned}
\zeta_{1 t}= & \left\{\sum_{j=0}^{\infty}\left[\mathbf{e}^{\prime} \mathbf{H}_{t+j, j}\left(\boldsymbol{\theta}_{0}\right) \mathbf{e}\right]^{2}\right\} \delta_{t}^{2} \\
& +2\left\{\sum_{j=0}^{\infty} \varepsilon_{t+j}\left[\mathbf{e}^{\prime} \mathbf{H}_{t+j, j}\left(\boldsymbol{\theta}_{0}\right) \mathbf{e}\right]\right\} \delta_{t}, \\
\zeta_{2 t}= & \left\{\sum_{j=0}^{\infty}\left[\mathbf{e}^{\prime} \mathbf{H}_{t+j, j}\left(\boldsymbol{\theta}_{0}\right) \mathbf{e}\right]^{2}\right\} \delta_{t}^{2} \\
& -2\left\{\sum_{j=0}^{\infty} \varepsilon_{t+j}\left[\mathbf{e}^{\prime} \mathbf{H}_{t+j, j}\left(\boldsymbol{\theta}_{0}\right) \mathbf{e}\right]\right\} \delta_{t}
\end{aligned}
$$

and

$$
\begin{aligned}
\delta_{t}= & \left(\phi_{100}-\phi_{200}\right)+\sum_{i=1}^{p}\left(\phi_{1 i 0}-\phi_{2 i 0}\right) y_{t-i} \\
& +\sum_{i=1}^{q}\left(\psi_{1 i 0}-\psi_{2 i 0}\right) \varepsilon_{t-i} .
\end{aligned}
$$

Let $F_{k}\left(\cdot \mid r_{0}\right)$ be the conditional distribution of $\zeta_{k, d+1}$ given $y_{1}=r_{0}$ for $k=1,2$. We define a two-sided compound Poisson process $\wp(z)$ as follows

$$
\wp(z)=\wp^{(1)}(-z) I(z<0)+\wp^{(2)}(z) I(z \geq 0),
$$

where $\left\{\wp^{(1)}(z), z \geq 0\right\}$ and $\left\{\wp^{(2)}(z), z \geq 0\right\}$ are two independent Poisson processes with $\wp^{(1)}(0)=\wp^{(2)}(0)=0$ a.s., with the same jump rate $\pi\left(r_{0}\right)>0$, where $\pi(x)$ is the density of $y_{1}$, and with the jump distributions $F_{1}\left(\cdot \mid r_{0}\right)$ and $F_{2}\left(\cdot \mid r_{0}\right)$, respectively. Clearly, $\wp(z)$ goes to $+\infty$ a.s. when $|z|$ tends to infinity since $\int x F_{k}\left(d x \mid r_{0}\right)>0$. Thus, there exists a unique random interval $\left[M_{-}, M_{+}\right)$on which the process $\{\wp(z), z \in R\}$ attains its global minimum a.s. Here, we work with the left continuous version for $\wp^{(1)}$ and the right continuous version for $\wp^{(2)}$. Now, we can state our other main result as follows:

Theorem 3.3. Suppose Assumptions 3.1-3.5 hold. Then $n\left(\widehat{r}_{n}-r_{0}\right) \stackrel{d}{\longrightarrow} M_{-}$and $n\left(\widehat{r}_{n}-r_{0}\right)$ is asymptotically independent of $\sqrt{n}\left(\widehat{\boldsymbol{\lambda}}_{n}-\boldsymbol{\lambda}_{0}\right)$ which is always asymptotically normally distributed.

Now, we give some remarks on (3.4) to end this section. When $q=0$, model (2.1) is a pure TAR model, that is, all $\psi_{i j}=0$. Thus, (3.4) reduces to $\zeta_{1 t}=\delta_{t}^{2}+2 \varepsilon_{t} \delta_{t}$ and $\zeta_{2 t}=$ $\delta_{t}^{2}-2 \varepsilon_{t} \delta_{t}$ with $\delta_{t}=\left(\phi_{100}-\phi_{200}\right)+\sum_{i=1}^{p}\left(\phi_{1 i 0}-\phi_{2 i 0}\right) y_{t-i}$. If the condition $y_{t-d}=r_{0}$ is given, then this coincides with the jump sizes of the compound Poisson process used in Chan (1993). When $p=0$, model (2.1) reduces to a pure TMA model and (3.4) does not change except for $\delta_{t}$ with $\delta_{t}=\left(\phi_{100}-\phi_{200}\right)+\sum_{i=1}^{q}\left(\psi_{1 i 0}-\psi_{2 i 0}\right) \varepsilon_{t-i}$. This is identical to those in $\mathrm{Li}$, Ling and $\mathrm{Li}$ (2010).

\section{NUMERICAL IMPLEMENTATION OF $M_{-}$}

In this section, we describe how to simulate the distribution of $M_{-}$when the order $\left(p_{1}, p_{2}, q_{1}, q_{2}\right)$ is known. From (3.5), we know that two factors determine the density of $M_{-}$, that is, the jump rate $\pi\left(r_{0}\right)$ and the jump distributions $F_{1}\left(\cdot \mid r_{0}\right)$ and $F_{2}\left(\cdot \mid r_{0}\right)$. We can simulate $M_{-}$via simulating the compound Poisson process (3.5) on the interval $[-T, T]$ for any given $T>0$ large enough since the expectations of the jumps are positive. Modifying Algorithm 6.2 in Cont and Tankov (2004, pp. 174) for a one-sided compound Poisson process, we have an algorithm for a two-sided compound Poisson process as follows:

\section{Algorithm B}

Step B.1. Generate two i.i.d. Poisson random variables $N_{1}$ and $N_{2}$ with the parameter $\pi\left(r_{0}\right) T$ as the total number of jumps on the intervals $[-T, 0]$ and $[0, T]$, respectively.

Step B.2. Given $N_{1}$ and $N_{2}$, generate $\left\{U_{1}, \ldots, U_{N_{1}}\right\}$ and $\left\{V_{1}, \ldots, V_{N_{2}}\right\}$ as two independent jump time sequences, where $\left\{U_{i}\right\} \stackrel{\text { i.i.d. }}{\sim} U[-T, 0]$ and $\left\{V_{i}\right\} \stackrel{\text { i.i.d. }}{\sim} U[0, T]$. Here, $U[a, b]$ denotes the uniform distribution on the interval $[a, b]$.

Step B.3. Given $N_{1}$ and $N_{2}$, generate $\left\{Y_{1}, \ldots, Y_{N_{1}}\right\}$ and $\left\{Z_{1}, \ldots, Z_{N_{2}}\right\}$ as two independent jump-size sequences from $F_{1}\left(\cdot \mid r_{0}\right)$ and $F_{2}\left(\cdot \mid r_{0}\right)$, respectively. 
For $z \in[-T, T]$, the trajectory of (3.5) is given by

$$
\begin{aligned}
\wp(z)= & I(z<0) \sum_{i=1}^{N_{1}} I\left(U_{i}>z\right) Y_{i} \\
& +I(z \geq 0) \sum_{j=1}^{N_{2}} I\left(V_{j}<z\right) Z_{j} .
\end{aligned}
$$

Then, we take the smallest minimizer of $\wp(z)$ on $[-T, T]$ as $M_{-}$. By repeating the above algorithm many times and using the nonparametric kernel method, we can get the density of $M_{-}$numerically.

In the above algorithm, the key is how to draw the jumpsize sequences $\left\{Y_{i}\right\}$ and $\left\{Z_{i}\right\}$ from $F_{1}\left(\cdot \mid r_{0}\right)$ and $F_{2}\left(\cdot \mid r_{0}\right)$ in Step B.3, respectively. Note that $\zeta_{k, d+1}$ 's in (3.4) are sums of infinite series and $\left\|\mathbf{H}_{d+1+j, j}\left(\boldsymbol{\theta}_{0}\right)\right\|=O\left(\rho^{j}\right)$ a.s. We truncate these infinite series and use the finite-term sums to approximate $\zeta_{k, d+1}$ 's as follows:

$\zeta_{k, d+1}^{(m)}$

$$
\begin{aligned}
= & \left\{\sum_{j=0}^{m}\left(\mathbf{e}^{\prime} \prod_{i=1}^{j}\left[\boldsymbol{\Psi}_{20}+\left(\boldsymbol{\Psi}_{10}-\boldsymbol{\Psi}_{20}\right) I\left(y_{i+1} \leq r_{0}\right)\right] \mathbf{e}\right)^{2}\right\} \delta_{d+1}^{2} \\
& +2(-1)^{k+1}\left\{\sum _ { j = 0 } ^ { m } \varepsilon _ { d + 1 + j } \left(\mathbf { e } ^ { \prime } \prod _ { i = 1 } ^ { j } \left[\boldsymbol{\Psi}_{20}+\left(\boldsymbol{\Psi}_{10}-\mathbf{\Psi}_{20}\right)\right.\right.\right. \\
& \left.\left.\left.\times I\left(y_{i+1} \leq r_{0}\right)\right] \mathbf{e}\right)\right\} \delta_{d+1},
\end{aligned}
$$

where $\boldsymbol{\Psi}_{i 0}$ is defined in (3.2) with $\psi_{i j}$ 's replaced by true values $\psi_{i j 0}$ 's, since the remaining terms are negligible when $m$ is large enough, where $\rho \in(0,1)$. Let $F_{k}^{(m)}\left(x \mid r_{0}\right)=P\left(\zeta_{k, d+1}^{(m)} \leq\right.$ $\left.x \mid y_{1}=r_{0}\right)$. Then, $F_{k}^{(m)}\left(x \mid r_{0}\right) \rightarrow F_{k}\left(x \mid r_{0}\right)$ as $m \rightarrow \infty$. In practice, the choice of $m$ is independent of the sample size $n$.

By the property of conditional expectation, the strong law of large numbers and $E\left[\pi\left(r_{0} \mid \mathbf{Z}_{0}\right)\right]=\pi\left(r_{0}\right)$, we have

$$
\begin{aligned}
& F_{k}^{(m)}\left(x \mid r_{0}\right) \\
& \quad=\int P\left(\zeta_{k, d+1}^{(m)} \leq x \mid y_{1}=r_{0}, \mathbf{z}_{0}=\mathbf{z}\right) \frac{\pi\left(r_{0} \mid \mathbf{z}\right)}{\pi\left(r_{0}\right)} G(d \mathbf{z}) \\
& \quad=\sum_{i=1}^{K} P\left(\zeta_{k, d+1}^{(m)} \leq x \mid y_{1}=r_{0}, \mathbf{z}_{0}=\mathbf{z}_{i}\right) \frac{\pi\left(r_{0} \mid \mathbf{z}_{i}\right)}{\sum_{l=1}^{K} \pi\left(r_{0} \mid \mathbf{z}_{l}\right)}+o(1) \\
& \quad \equiv F_{k, K}^{(m)}\left(x \mid r_{0},\left\{\mathbf{z}_{j}\right\}\right)+o(1) \quad \text { a.s. }
\end{aligned}
$$

uniformly in $x \in \mathbb{R}$ by Theorem 2 in Pollard (1984, page 8), where $\mathbf{Z}_{0}=\left(y_{0}, \ldots, y_{1-(p \vee d)}, \varepsilon_{0}, \ldots, \varepsilon_{1-q}\right)^{\prime}, \mathbf{z}_{i} \in R^{(p \vee d)+q}$, $G(\cdot)$ is the distribution of $\mathbf{Z}_{0}, \pi\left(r_{0} \mid \mathbf{z}\right)$ is the conditional density of $y_{1}$ given $\mathbf{Z}_{0}=\mathbf{z}$. Here, the choices of $m$ and $K$ are independent.
Denote

$h\left(\mathbf{Z}_{t-1}, \boldsymbol{\theta}\right)= \begin{cases}\phi_{10}+\sum_{i=1}^{p_{1}} \phi_{1 i} y_{t-i}+\sum_{i=1}^{q_{1}} \psi_{1 i} \varepsilon_{t-i}, & \text { if } y_{t-d} \leq r, \\ \phi_{20}+\sum_{i=1}^{p_{2}} \phi_{2 i} y_{t-i}+\sum_{i=1}^{q_{2}} \psi_{2 i} \varepsilon_{t-i}, & \text { if } y_{t-d}>r .\end{cases}$

Then, model (2.1) can be written as $y_{t}=\varepsilon_{t}+h\left(\mathbf{Z}_{t-1}, \boldsymbol{\theta}_{0}\right)$. Given $y_{1}=r_{0}$ and $\mathbf{Z}_{0}=\mathbf{z}$, we have $\varepsilon_{1}=r_{0}-h\left(\mathbf{z}, \boldsymbol{\theta}_{0}\right)$ and $\pi\left(r_{0} \mid \mathbf{z}\right)=f_{\varepsilon}\left(r_{0}-h\left(\mathbf{z}, \boldsymbol{\theta}_{0}\right)\right)$.

Let $F_{\varepsilon}(\cdot)$ be the cumulative distribution function of $\varepsilon_{t}$. When $\boldsymbol{\theta}_{0}, \pi\left(r_{0}\right), F_{\varepsilon}(\cdot)$ and $G(\cdot)$ are known, the following algorithm describes how to sample $Y_{1}$ from $F_{k, K}^{(m)}\left(x \mid r_{0},\left\{\mathbf{z}_{j}\right\}\right)$.

\section{Algorithm C}

Step C.1. Draw a sample $\mathcal{Z}=\left\{\mathbf{z}_{1}, \ldots, \mathbf{z}_{K}\right\}$ from $G(\cdot)$.

Step C.2. For each $i \in\{1, \ldots, K\}$, sample independently $\left\{\varepsilon_{t}: 2 \leq t \leq d+1+m\right\}$ from $F_{\varepsilon}(\cdot)$ and generate $\left\{y_{t}: 2 \leq t \leq d+1+m\right\}$ by iterating model (2.1) with the initial value $y_{1}=r_{0}, \mathbf{Z}_{0}=\mathbf{z}_{i}$ and $\varepsilon_{1}=r_{0}-h\left(\mathbf{z}_{i}, \boldsymbol{\theta}_{0}\right)$. Then calculate $\zeta_{1, d+1}^{(m)}$, denoted by $\xi_{1, d+1}^{(m, i)}$.

Step C.3. Calculate $\pi\left(r_{0} \mid \mathbf{z}_{i}\right)^{\prime}$ s and sample a $U$ from the conditional discrete density: $P(U=i \mid \mathcal{Z})=$ $\pi\left(r_{0} \mid \mathbf{z}_{i}\right) /\left[\sum_{l=1}^{K} \pi\left(r_{0} \mid \mathbf{z}_{l}\right)\right]$ for $i=1, \ldots, K$, independent of all $\left\{\varepsilon_{t}, t \geq 2\right\}$.

Step C.4. Obtain $Y_{1}=\xi_{1, d+1}^{(m, U)}$.

Clearly, by Step C.2 and Step C.3, $\left\{y_{t}, t \geq 2\right\}$ is independent of $U$ given $\mathcal{Z}$, and so is $\xi_{1, d+1}^{(m, i)}$. Denote the conditional measure $P_{\mathcal{Z}}(\cdot \mid A) \equiv P(\cdot \mid A, \mathcal{Z})$. Thus,

$$
\begin{aligned}
P_{\mathcal{Z}} & \left(Y_{1} \leq x\right) \\
& =\sum_{i=1}^{K} P_{\mathcal{Z}}\left(\xi_{1, d+1}^{(m, i)} \leq x, U=i\right) \\
& =\sum_{i=1}^{K} P_{\mathcal{Z}}\left(\xi_{1, d+1}^{(m, i)} \leq x\right) P_{\mathcal{Z}}(U=i) \\
& =\sum_{i=1}^{K} P\left(\zeta_{1, d+1}^{(m)} \leq x \mid y_{1}=r_{0}, \mathbf{z}_{0}=\mathbf{z}_{i}\right) \frac{\pi\left(r_{0} \mid \mathbf{z}_{i}\right)}{\sum_{l=1}^{K} \pi\left(r_{0} \mid \mathbf{z}_{l}\right)} \\
& =F_{1, K}^{(m)}\left(x \mid r_{0},\left\{\mathbf{z}_{j}\right\}\right) .
\end{aligned}
$$

Since $F_{k, K}^{(m)}\left(x \mid r_{0},\left\{\mathbf{z}_{j}\right\}\right) \stackrel{K \rightarrow \infty}{\longrightarrow} F_{k}^{(m)}\left(x \mid r_{0}\right) \stackrel{m \rightarrow \infty}{\longrightarrow} F_{k}\left(x \mid r_{0}\right)$, $Y_{1} \mid \mathcal{Z} \sim F_{1}\left(x \mid r_{0}\right)$ asymptotically. Denote the sequences of the two-sided CPPs by $\left\{\wp^{(m)}(z): z \in R\right\}$ and $\left\{\wp_{K}^{(m)}(z): z \in R\right\}$ which are determined by the same jump rate $\pi\left(r_{0}\right)$ and jump distributions $F_{k}^{(m)}\left(x \mid r_{0}\right)$ and $F_{k, K}^{(m)}\left(x \mid r_{0},\left\{\mathbf{z}_{j}\right\}\right)$, respectively. By Theorem 16 in Pollard (1984, page 134), we have a.s. that $\wp_{K}^{(m)}(z) \stackrel{K \rightarrow \infty}{\Longrightarrow} \wp^{(m)}(z) \stackrel{m \rightarrow \infty}{\Longrightarrow} \wp(z)$ in $D(R)$. Minimizing the process $\wp_{K}^{(m)}(z)$, we can get the smallest minimizer $\widetilde{M}_{K}^{(m)}$. By Theorem 3.1 (on the continuity of the smallest argmax functional) in Seijo and Sen (2011), $\widetilde{M}_{K}^{(m)} \Longrightarrow M_{-}$(first $K \rightarrow \infty$, then $m \rightarrow \infty)$. Summarizing the above discussion, we have 
Theorem 4.1. Suppose Assumptions 3.1-3.2 hold and $\left\{y_{t}\right\}$ is strictly stationary and ergodic. Then, almost surely,

$$
\lim _{m \rightarrow \infty} \lim _{K \rightarrow \infty}\left|P_{\mathcal{Z}}\left(\widetilde{M}_{K}^{(m)} \leq x\right)-P\left(M_{-} \leq x\right)\right|=0
$$

at each $x$ for which $P\left(M_{-}=x\right)=0$.

Since $\widetilde{M}_{K}^{(m)}$ is only relevant to model (2.1) and independent of the estimation of the parameter, without loss of generality, in what follows, we regard $\widetilde{M}_{K}^{(m)}$ as $M_{-}$.

In practice, however, since only one sample $\mathfrak{X} \equiv$ $\left\{y_{1}, \ldots, y_{n}\right\}$ is available, we can use it to estimate $\boldsymbol{\theta}_{0}$ and $\pi\left(r_{0}\right)$ a.s., denoting the estimators as $\widehat{\boldsymbol{\theta}}_{n}$ and $\widehat{\pi}\left(\widehat{r}_{n}\right)$, respectively, where $\widehat{\pi}(\cdot)$ is the kernel density estimator of $\pi(\cdot)$, and calculate the residuals $\left\{\widehat{\varepsilon}_{t}: k_{0}+1 \leq t \leq n\right\}$, where $k_{0}=\max (p, d, q)$. Based on the residuals, we construct the estimators $\widehat{F}_{\varepsilon}(\cdot)$ and $\widehat{f}_{\varepsilon}(\cdot)$ of $F_{\varepsilon}(\cdot)$ and $f_{\varepsilon}(\cdot)$ as follows

$$
\widehat{F}_{\varepsilon}(x)=\frac{1}{n-k_{0}} \sum_{t=k_{0}+1}^{n}\left(\varepsilon_{t}^{*} \leq x\right),
$$

where $\varepsilon_{t}^{*}=\widehat{\varepsilon}_{t}-\overline{\hat{\varepsilon}}$ with $\overline{\hat{\varepsilon}}=\frac{1}{n-k_{0}} \sum_{t=k_{0}+1}^{n} \widehat{\varepsilon}_{t}$, and

$$
\widehat{f}_{\varepsilon}(x)=\frac{1}{n-k_{0}} \sum_{t=k_{0}+1}^{n} \frac{1}{\widehat{h}_{\mathrm{opt}}^{*}} K\left(\frac{\varepsilon_{t}^{*}-x}{\widehat{h}_{\mathrm{opt}}^{*}}\right)
$$

where $K(x)=(\sqrt{2 \pi})^{-1} \exp \left(-x^{2} / 2\right)$ is the Gaussian kernel and $\widehat{h}_{\mathrm{opt}}^{*}$ is the bandwidth, which can be selected by

$$
\widehat{h}_{\mathrm{opt}}^{*}=\widehat{h}_{\mathrm{opt}}\left(1+\frac{35}{48} \widehat{\gamma}_{4}+\frac{35}{32} \widehat{\gamma}_{3}^{2}+\frac{385}{1024} \widehat{\gamma}_{4}^{2}\right)^{-1 / 5}
$$

where $\widehat{h}_{\text {opt }}=1.06 s\left(n-k_{0}\right)^{-1 / 5}$ is the reference bandwidth selector, and $s, \widehat{\gamma}_{3}$ and $\widehat{\gamma}_{4}$ are the sample standard deviation, skewness and kurtosis of the residuals $\left\{\widehat{\varepsilon}_{t}: k_{0}+1 \leq t \leq n\right\}$, respectively. See Fan and Yao (2003, pp. 201). Of course, one can use other kernel functions and bandwidths. When $f_{\varepsilon}(\cdot)$ is uniformly continuous, using the technique in Silverman (1978), we can show that $\sup _{x \in R}\left|\widehat{f}_{\varepsilon}(x)-f_{\varepsilon}(x)\right|=o_{p}(1)$ as $n \rightarrow \infty$. The following algorithm is just an extension of Algorithm C under $\widehat{\varepsilon}_{t}$ and $\widehat{\pi}\left(\widehat{r}_{n}\right)$.

\section{Algorithm D}

Step D.1. Set $\hat{\mathbf{z}}_{i}=\left(y_{i}, \ldots, y_{i-(p \vee d)+1}, \widehat{\varepsilon}_{i}, \ldots, \widehat{\varepsilon}_{i-q+1}\right)^{\prime}$ for $i=k_{0}+1, \ldots, n$.

Step D.2. For each $i \in\left\{k_{0}+1, \ldots, n\right\}$, sample independently $\left\{\tilde{\varepsilon}_{t}: 2 \leq t \leq d+1+m\right\}$ from $\widehat{F}_{\varepsilon}$ given $\mathfrak{X}$ and generate $\left\{\tilde{y}_{t}: 2 \leq t \leq d+1+m\right\}$ by iterating model (2.1) with the initial value $y_{1}=\widehat{r}_{n}, \mathbf{Z}_{0}=\hat{\mathbf{z}}_{i}$ and $\varepsilon_{1}=\widehat{r}_{n}-h\left(\hat{\mathbf{z}}_{i}, \widehat{\boldsymbol{\theta}}_{n}\right)$ and $\boldsymbol{\theta}_{0}$ being replaced by $\widehat{\boldsymbol{\theta}}_{n}$. Then calculate $\tilde{\zeta}_{1, d+1}^{(m)}$, denoted by $\tilde{\xi}_{1, d+1}^{(m, i)}$, where

$$
\begin{aligned}
\tilde{\zeta}_{1, d+1}^{(m)}= & \left\{\sum _ { j = 0 } ^ { m } \left(\mathbf { e } ^ { \prime } \prod _ { i = 1 } ^ { j } \left[\widehat{\mathbf{\Psi}}_{2 n}+\left(\widehat{\mathbf{\Psi}}_{1 n}-\widehat{\mathbf{\Psi}}_{2 n}\right)\right.\right.\right. \\
& \left.\left.\left.\times I\left(\tilde{y}_{i+1} \leq \widehat{r}_{n}\right)\right] \mathbf{e}\right)^{2}\right\} \delta_{d+1}^{* 2} \\
& +2\left\{\sum _ { j = 0 } ^ { m } \tilde { \varepsilon } _ { d + 1 + j } \left(\mathbf { e } ^ { \prime } \prod _ { i = 1 } ^ { j } \left[\widehat{\mathbf{\Psi}}_{2 n}+\left(\widehat{\mathbf{\Psi}}_{1 n}-\widehat{\mathbf{\Psi}}_{2 n}\right)\right.\right.\right. \\
& \left.\left.\left.\times I\left(\tilde{y}_{i+1} \leq \widehat{r}_{n}\right)\right] \mathbf{e}\right)\right\} \delta_{d+1}^{*}
\end{aligned}
$$

and

$$
\begin{aligned}
\delta_{d+1}^{*}= & \left(\widehat{\phi}_{10 n}-\widehat{\phi}_{20 n}\right)+\sum_{s=1}^{p}\left(\widehat{\phi}_{1 s n}-\widehat{\phi}_{2 s n}\right) y_{d+1-s}^{*} \\
& +\sum_{s=1}^{q}\left(\widehat{\psi}_{1 s n}-\widehat{\psi}_{2 s n}\right) \epsilon_{d+1-s}^{*}
\end{aligned}
$$

with

$$
y_{j}^{*}= \begin{cases}\tilde{y}_{j}, & \text { if } j \geq 2 \\ \widehat{r}_{n}, & \text { if } j=1 \\ y_{i+j}, & \text { if } j \leq 0\end{cases}
$$

and

$$
\epsilon_{j}^{*}= \begin{cases}\tilde{\varepsilon}_{j}, & \text { if } j \geq 2, \\ \widehat{r}_{n}-h\left(\hat{\mathbf{z}}_{i}, \widehat{\boldsymbol{\theta}}_{n}\right), & \text { if } j=1, \\ \widehat{\varepsilon}_{i+j}, & \text { if } j \leq 0 .\end{cases}
$$

Step D.3. Calculate $\widehat{\pi}\left(\widehat{r}_{n} \mid \hat{\mathbf{z}}_{i}\right)$ 's and sample a $U$ from the conditional discrete density: $P(U=i \mid \mathfrak{X})=\widehat{\pi}\left(\widehat{r}_{n} \mid \hat{\mathbf{z}}_{i}\right) /$ $\left[\sum_{l=k_{0}+1}^{n} \widehat{\pi}\left(\widehat{r}_{n} \mid \hat{\mathbf{z}}_{l}\right)\right]$ for $i=k_{0}+1, \ldots, n$, conditionally independent of $\left\{\tilde{\varepsilon}_{t}, t \geq 2\right\}$ given $\mathfrak{X}$.

Step D.4. Obtain $\tilde{Y}_{1}=\tilde{\xi}_{1, d+1}^{(m, U)}$.

Denote by $\widehat{M}_{n}^{(m)}$ the counterpart of $\widetilde{M}_{K}^{(m)}$ obtained using Algorithm D. By the same procedure used in the proof of Theorem 3.2 in Li, Ling and Li (2010), we have

Theorem 4.2. Suppose Assumptions 3.1-3.5 hold and $f_{\varepsilon}(x)$ is uniformly continuous on $R$. Then, in probability,

$$
\lim _{m \rightarrow \infty} \lim _{n \rightarrow \infty}\left|P_{\mathfrak{X}}\left(\widehat{M}_{n}^{(m)} \leq x\right)-P\left(M_{-} \leq x\right)\right|=0
$$

at each $x$ for which $P\left(M_{-}=x\right)=0$.

We now consider the following TARMA $(1,1,1,1)$ model:

$$
y_{t}= \begin{cases}\phi_{10}+\phi_{11} y_{t-1}+\varepsilon_{t}+\psi_{11} \varepsilon_{t-1}, & \text { if } y_{t-1} \leq r_{0} \\ \phi_{20}+\phi_{21} y_{t-1}+\varepsilon_{t}+\psi_{21} \varepsilon_{t-1}, & \text { if } y_{t-1}>r_{0}\end{cases}
$$

where $\boldsymbol{\theta}_{0}=\left(\phi_{10}, \phi_{11}, \psi_{11}, \phi_{20}, \phi_{21}, \psi_{21}, r_{0}\right)^{\prime}=(0.6,0.6$, $-0.7,-1,0.4,0.5,0.2)^{\prime}$ and $\left\{\varepsilon_{t}\right\}$ is i.i.d standard normal. 

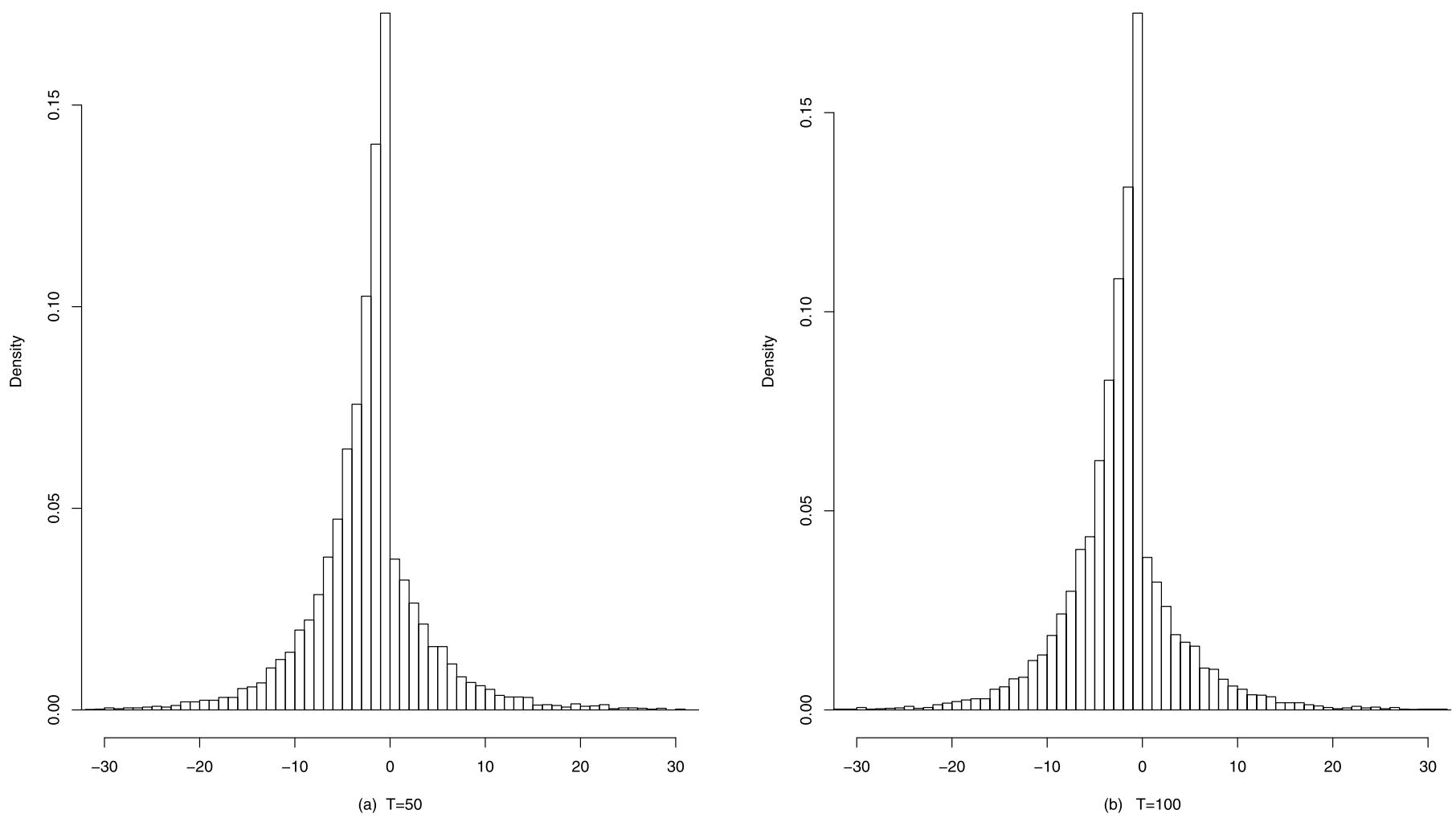

Figure 1. The density of $M_{-}$for different values of $T$. (a) $T=50$; (b) $T=100$.

Table 1. Quantiles for $M_{-}$under model (4.3)

\begin{tabular}{lllllllll}
\hline$\alpha$ & $0.5 \%$ & $1 \%$ & $2.5 \%$ & $5 \%$ & $95 \%$ & $97.5 \%$ & $99 \%$ & $99.5 \%$
\end{tabular}

\begin{tabular}{lllllllll}
$Q$ & -22.05 & -19.18 & -14.74 & -11.61 & 7.27 & 10.88 & 15.86 & 20.06 \\
\hline
\end{tabular}

Since it is difficult to obtain the exact value of $\pi\left(r_{0}\right)$, we adopt the kernel method to estimate it, where the sample size is $N=10,000$ and the Gaussian kernel is used. The bandwidth is chosen by (4.2). To ensure the precision of the estimation, 100 replications are used. Finally, we get $\pi\left(r_{0}\right)=0.3241$.

Figure 1 gives the densities of $M_{-}$when $T=50$ and 100 by using Algorithms B and C. 1,001 observations (i.e., $K=1,000)$ together with the driven noises generated by model (4.3) and $m=200$ are used to calculate the jump sizes $\zeta_{k, d+1}^{(m)}, k=1,2$. Then, minimizing the process (4.1), we can obtain an observation of $M_{-}$. Here, 10,000 replications are used to get the density of $M_{-}$. From Fig 1, we can see that the densities of $M_{-}$are very close to each other for $T=50$ and 100 . In theory, the larger $T$, the more precise the density of $M_{-}$. How to select a suitable $T$ is an interesting and open problem and there is no theory to support this in the literature.

Table 1 gives the quantiles of $M_{-}$under model (4.3) when the significance level $\alpha=0.005,0.01,0.025,0.05,0.95,0.975$, 0.99 and 0.995 .
For Algorithm D, simulation studies show that it works well in practice. Suppose that a sample $\left\{y_{1}, \ldots, y_{400}\right\}$ is from model (4.3) and fixed. Then we consistently estimate the quantities involved in Algorithm D. $m=200$ and 1,000 replications are used to obtain $\widehat{M}_{n}^{(m)}$. Fig 2 (a) shows the empirical cumulative distribution functions of $\widehat{M}_{n}^{(m)}$ and $\widetilde{M}_{K}^{(m)}$. Figure 2 (b) gives the density of $\widehat{M}_{n}^{(m)}$, which is close to those in Fig 1.

To further evaluate the performance of the simulation method above, we consider another example, e.g., a higherorder TARMA $(3,3,0,0)$ model:

$$
y_{t}= \begin{cases}1+0.2 y_{t-1}-0.4 y_{t-2}+0.3 y_{t-3}+\varepsilon_{t}, & y_{t-3} \leq 0 \\ -1+0.6 y_{t-1}+0.1 y_{t-2}-0.2 y_{t-3}+\varepsilon_{t}, & y_{t-3}>0\end{cases}
$$

where $\varepsilon_{t} \sim$ i.i.d. $\mathcal{N}(0,1)$. The simulation results are displayed in Fig 3. The density of $n\left(\widehat{r}_{n}-r_{0}\right)$ is given in Fig 3 (a), where the sample size is 600 and 10,000 replications are used. Figure 3 (b) shows the density of $M_{-}$, where 10,000 replications are used. Figures 3 (c)-(d) show the density of $\widehat{M}_{-}$(obtained by Algorithms $\mathrm{B}$ and $\mathrm{D}$ ) for a given and fixed sample $\left\{y_{1}, \ldots, y_{600}\right\}$ from model (4.4), where 1,000 and 10,000 replications are used for (c) and (d), respectively. From Fig 3, we can see that (a), (b) and (d) are very matching. 

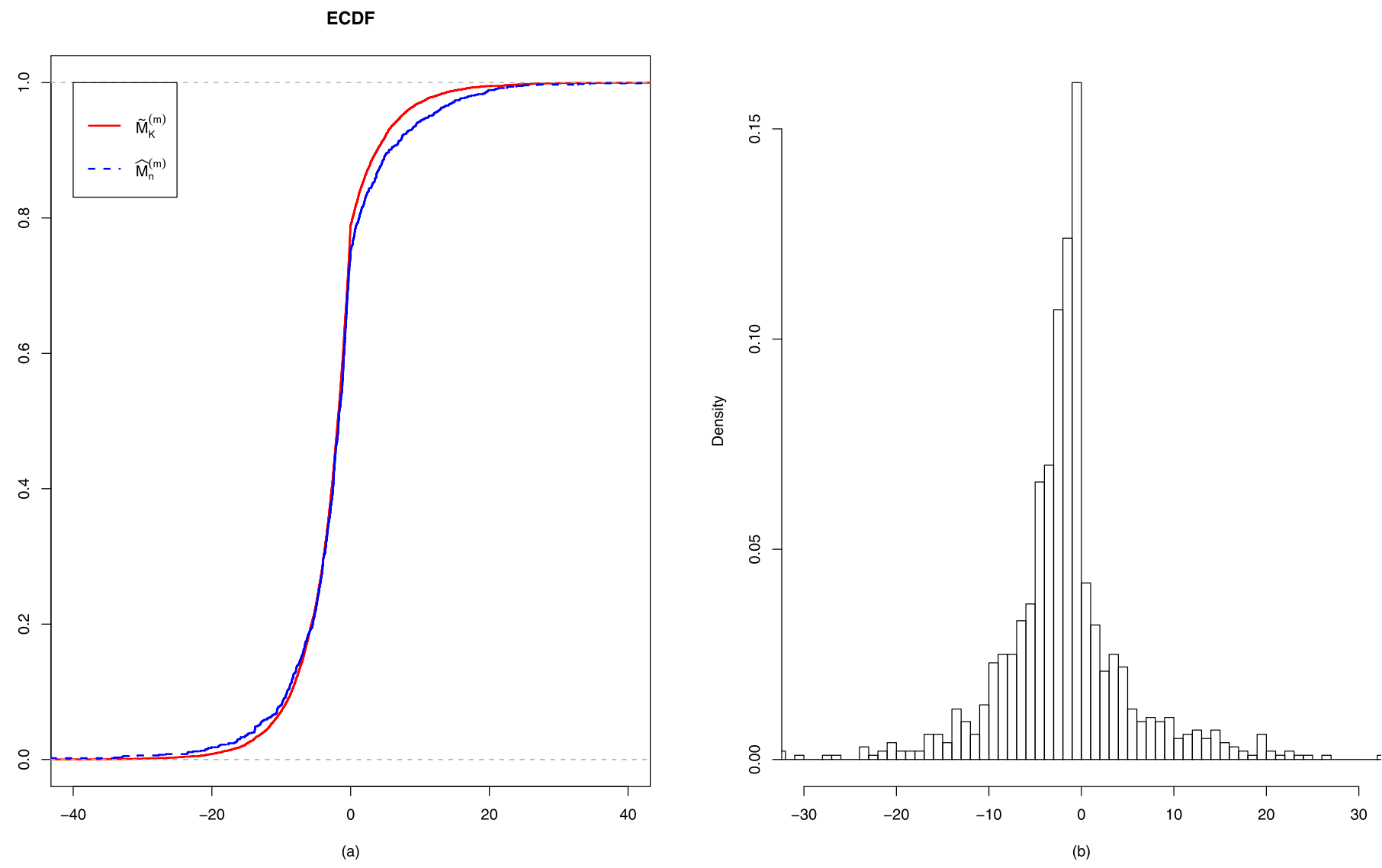

Figure 2. (a) The empirical cumulative distribution functions of $\widetilde{M}_{K}^{(m)}\left(M_{-}\right)$and $\widehat{M}_{n}^{(m)}$. (b) The density of $\widehat{M}_{n}^{(m)}$, where the sample size is $n=400$ and 1,000 replications are used.

\section{SIMULATION STUDIES}

To assess the performance of the LSE of $\boldsymbol{\theta}_{0}$ in finite samples, we use sample sizes $n=200,400,600$ and 800 , each with replications 1,000 for model (4.3) with $\varepsilon_{t} \sim \mathcal{N}(0,1)$. In Table 2, we summarize the empirical mean, empirical standard deviation (ESD) and asymptotic standard deviation (ASD). Here, the ASD of $\widehat{\boldsymbol{\lambda}}$ are computed using $\Sigma$ in Theorem 3.2 and the ASD of $\widehat{r}_{n}$ is obtained by simulating $M_{-}$.

From Table 2, we see that the consistency of the estimators is shown by the empirical means and the closeness of the empirical standard deviations to the asymptotic standard deviations. We also see that the values of the ESDs for $\widehat{r}_{n}$ are about halved each time when the value of $n$ is doubled. This partially illustrates the $n$-consistency of the threshold estimator, under which the estimator of the threshold parameter would approach the true parameter much faster than the coefficient parameter estimators.

Table 3 reports the coverage probabilities of the estimator $\widehat{r}_{n}$ for $n=200,400,600$ and 800, respectively, based on the critical values in Table 1 . From the table, we can see that
Table 2. Simulation studies for model (4.3) with

$$
\boldsymbol{\theta}_{0}=(0.6,0.6,-0.7,-1,0.4,0.5,0.2)^{\prime}
$$

\begin{tabular}{|c|c|c|c|c|c|c|c|c|}
\hline$n$ & & $\phi_{10}$ & $\phi_{11}$ & $\psi_{11}$ & $\phi_{20}$ & $\phi_{21}$ & $\psi_{21}$ & $r$ \\
\hline \multirow[t]{3}{*}{200} & EM & 0.6149 & 0.6099 & -0.7241 & -1.0063 & 0.4046 & 0.5107 & 0.1877 \\
\hline & ESD & 0.1315 & 0.1920 & 0.1935 & 0.2103 & 0.2101 & 0.1936 & 0.0325 \\
\hline & ASD & 0.11 & 0.1 & 0.1553 & 0.2 & o & & 0.02 \\
\hline \multirow[t]{3}{*}{400} & $\mathrm{EM}$ & 0.60 & 0.6047 & -0.7092 & -1.0 & & & 0. \\
\hline & ESD & 0.0 & 0.1 & 0.1226 & 0. & 6 & 0 & 0.0 \\
\hline & ASD & 0.08 & 0.1157 & 0.1098 & 0.1416 & 0.1299 & 0.1 & 0.0148 \\
\hline \multirow[t]{3}{*}{600} & $\mathrm{EM}$ & 0.60 & .6059 & -0.7071 & -1.0 & & & \\
\hline & ESD & 0.06 & 0.0952 & 0.0 & & & & 0.0093 \\
\hline & ASD & 0.06 & & 0.0897 & 0.1156 & & & 0.0099 \\
\hline \multirow[t]{3}{*}{800} & EM & 0.599 & 60 & -0.7 & -1 . & & & 19 \\
\hline & $\mathrm{E}$ & & & & & & & 771 \\
\hline & ASD & 0.05 & 0.0818 & 0.0777 & 0.1 & 0.09 & 0.076 & 0.0074 \\
\hline
\end{tabular}

the coverage probability is rather accurate even though the sample size $n$ is 200 .

Figure 4 shows the density of $n\left(\widehat{r}_{n}-r_{0}\right)$ when the sample size $n=200,400,600$ and 800, respectively. From Fig 4, we see that the densities of $n\left(\widehat{r}_{n}-r_{0}\right)$ and $M_{-}$in Fig 1 


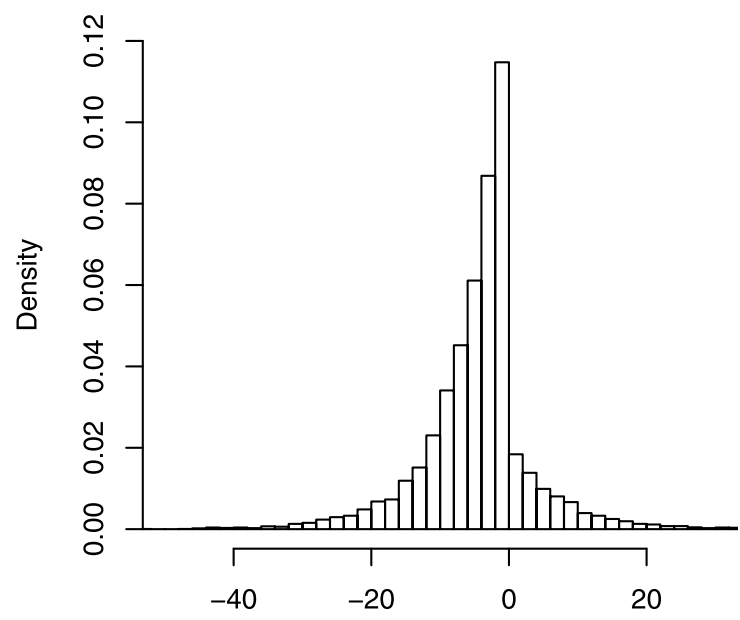

(a)

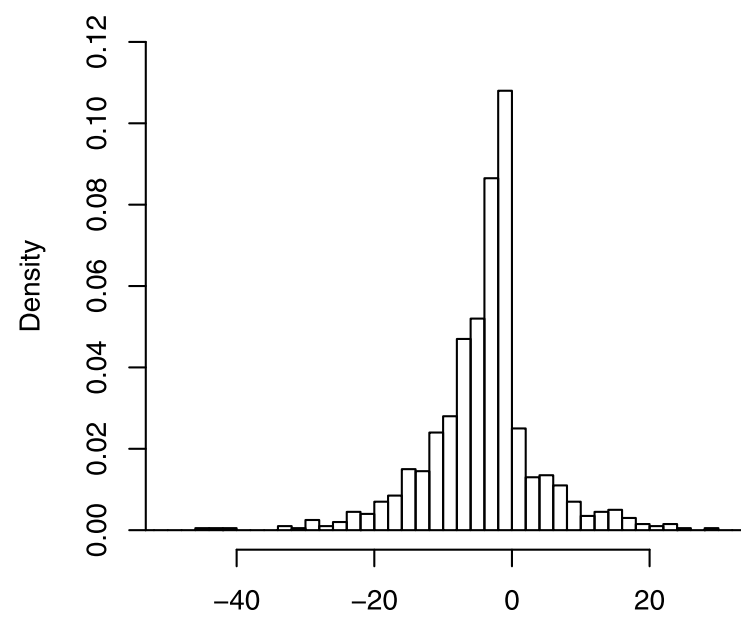

(c)

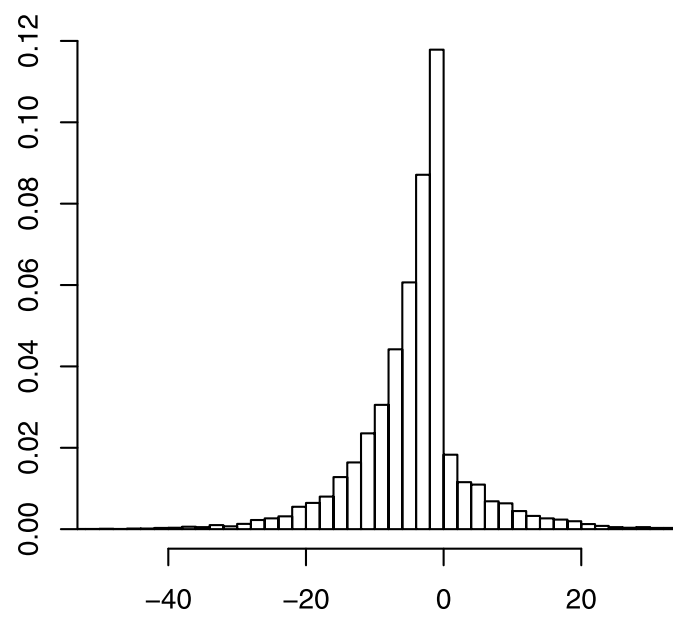

(b)

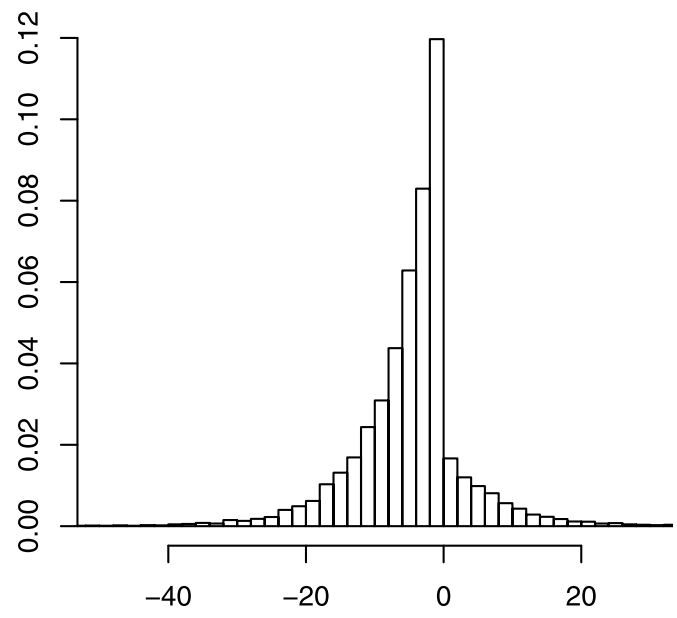

(d)

Figure 3. (a) The density of $n\left(\widehat{r}_{n}-r_{0}\right)$. (b) The density of $M_{-}$. (c) The density of $\widehat{M}_{-}$when 1,000 replications are used. (d) The density of $\widehat{M}_{-}$when 10,000 replications are used.

Table 3. Coverage probabilities

\begin{tabular}{lcccc}
\hline$\alpha$ & 200 & 400 & 600 & 800 \\
\hline 0.01 & 0.979 & 0.986 & 0.991 & 0.988 \\
0.05 & 0.936 & 0.936 & 0.966 & 0.960 \\
0.10 & 0.890 & 0.888 & 0.917 & 0.911 \\
\hline
\end{tabular}

are matching, which supports Theorem 3.3 empirically. We also see that the density of $n\left(\widehat{r}_{n}-r_{0}\right)$ is leptokurtic and asymmetric. Here the skewness of $M_{-}$is 0.234 and the kurtosis is 8.96. Due to the skewness and kurtosis, we must be careful in constructing confidence intervals of the estimated threshold in practice.

\section{CONCLUDING REMARKS}

This paper considers the general TARMA model which contains the pure TAR model and the pure TMA model as two special cases and has established the asymptotic theory of the least squares estimation. The limiting distribution of the estimated threshold, which is the smallest minimizer of a two-sided compound Poisson process, has been derived. A numerical method is proposed to simulate this limiting distribution and simulation studies show that it does work well. These results are obtained under which the order $\left(p_{1}, p_{2}, q_{1}, q_{2}\right)$ is known. In applications, one can use either the AIC or BIC to determine the unknown order. Here we do not pursue this issue anymore. As for TARMA model (3.3) 


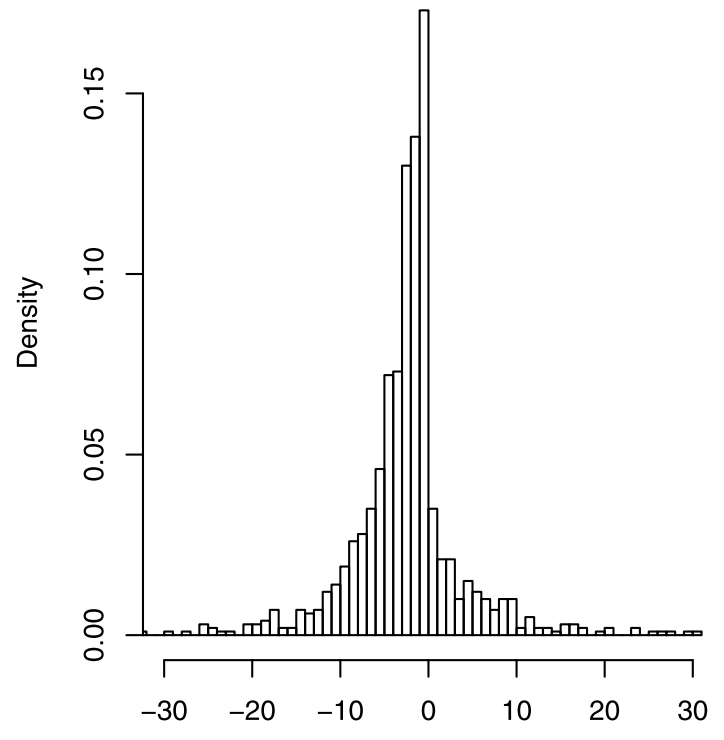

(a) $n=200$

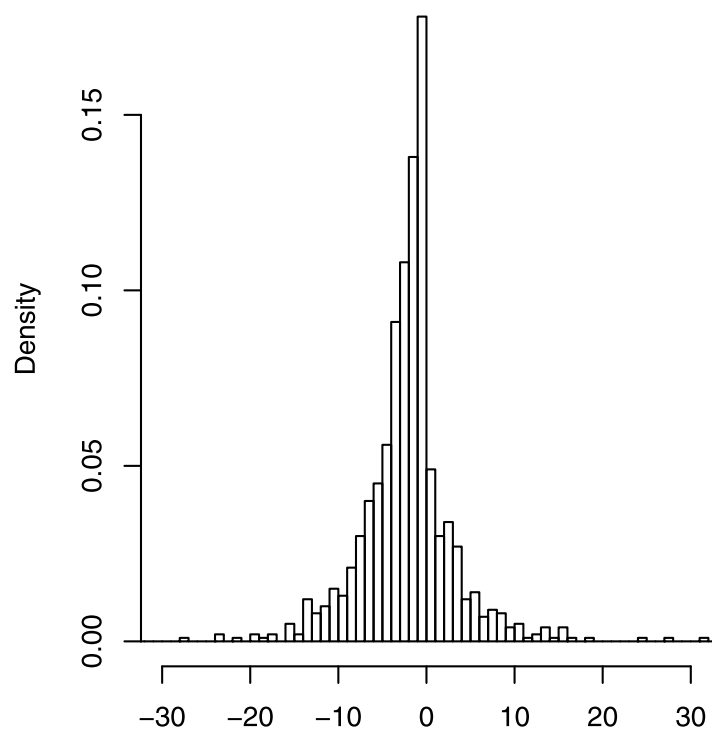

(c) $n=600$

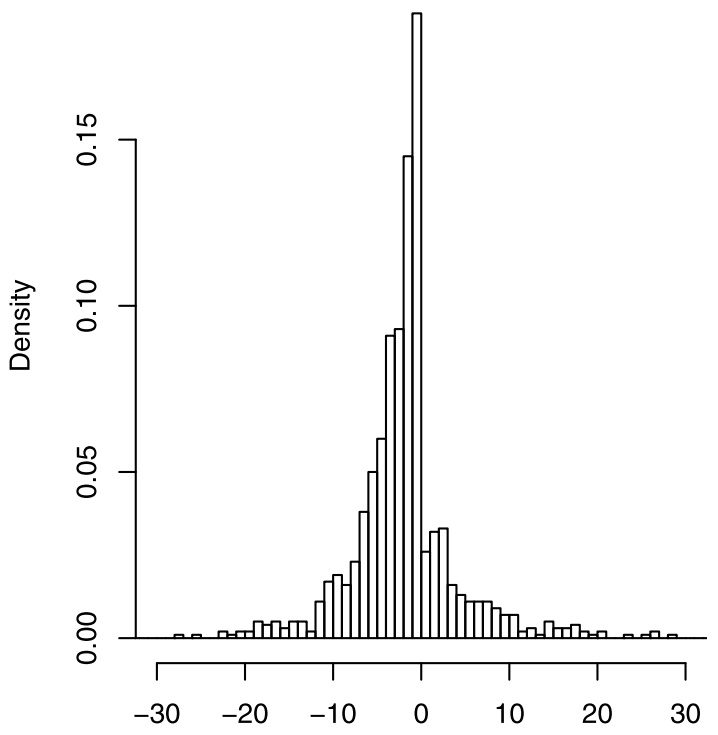

(b) $n=400$

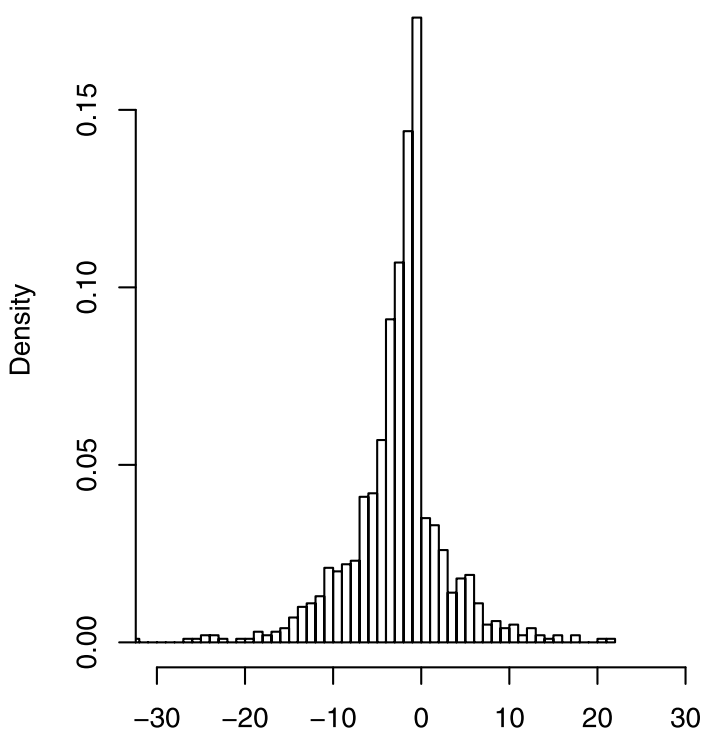

(d) $n=800$

Figure 4. The density of $n\left(\widehat{r}_{n}-r_{0}\right)$ when the sample size $n=200,400,600$ and 800, respectively.

where the autoregressive function is continuous and where there is no threshold in the moving-average part, we conjecture that the estimated threshold has a $\sqrt{n}$-convergence rate and is asymptotically normal as well as other parameters. This conjecture corresponds to the results in Chan and Tsay (1998) on TAR models when the autoregressive function is continuous. For this case, further study will be needed in the future.

\section{APPENDIX A. PROOF OF THEOREM 3.2}

In this section we give the proof for the case $q=1$ and $1 \leq d \leq p$. For a general case, the proof is similar. We first give a lemma.

Lemma A.1. Suppose that Assumptions 3.1-3.5 hold. Then, for any $\varepsilon>0$ and $\eta>0$, there exists a constant 
$B>0$ such that for any fixed $\delta \in(0,1)$ small enough and any $n \geq 1$

$$
\begin{aligned}
& P\left(\sup _{B / n<u<\delta}\left|\frac{\sum_{t=1}^{n} I^{*}\left(r_{0}<y_{t-d} \leq r_{0}+u\right)}{n G^{*}(u)}-1\right|<\eta\right) \\
& \quad>1-\varepsilon, \\
& P\left(\sup _{B / n<u<\delta}\left|\frac{\sum_{t=1}^{n} A_{t}(0, u)}{n G(u)}\right|<\eta\right)>1-\varepsilon, \\
& P\left(\sup _{B / n<u<\delta}\left|\frac{\sum_{t=1}^{n} \varepsilon_{t}\left[\varepsilon_{t}\left(\boldsymbol{\lambda}_{0}, r_{0}+u\right)-\varepsilon_{t}\right]}{n G(u)}\right|<\eta\right) \\
& \quad>1-\varepsilon,
\end{aligned}
$$

where $I^{*}\left(r_{0}<y_{t-d} \leq r\right)=I\left(r_{0}<y_{t-d} \leq r,\left\|\mathbf{Z}_{t-1}-\mathbf{Z}^{*}\right\| \leq\right.$ $\beta), G^{*}(u)=P\left(r_{0}<y_{t-d} \leq r_{0}+u,\left\|\mathbf{Z}_{t-1}-\mathbf{Z}^{*}\right\| \leq\right.$ $\beta), G(u)=P\left(r_{0}<y_{t-d} \leq r_{0}+u\right)$ and $A_{t}\left(u_{1}, u_{2}\right)=$ $I\left(r_{0}+u_{1}<y_{t-d} \leq r_{0}+u_{2}\right) \sum_{j=0}^{\infty} \rho^{j}\left\|\mathbf{Z}_{t-2-j}\right\| I\left(r_{0}+u_{1}<\right.$ $\left.y_{t-1-d-j} \leq r_{0}+u_{2}\right)$ for $u, u_{1}, u_{2} \in[0, \delta]$. Here $\beta$ is a fixed positive constant and $\mathbf{Z}^{*}=\left(\mathbf{w}^{\prime}, \mathbf{a}^{\prime}\right)^{\prime}$ defined in Assumption 3.5.

Proof. The proof of (A.1) is simple by Assumption 3.4, similar to that of (4.4a) in Chan (1993) and hence it is omitted. Since the proof of (A.3) is similar to that of (A.2), we only prove (A.2). It is sufficient to verify the following inequalities for $0 \leq u_{1}, u_{2} \leq \delta$

$$
\begin{aligned}
& E\left[A_{t}\left(u_{1}, u_{2}\right)\right] \leq C\left[G\left(u_{2}\right)-G\left(u_{1}\right)\right], \\
& \operatorname{Var}\left(A_{t}\left(u_{1}, u_{2}\right)\right) \leq C\left[G\left(u_{2}\right)-G\left(u_{1}\right)\right], \\
& \operatorname{Var}\left(\sum_{t=1}^{n} A_{t}\left(u_{1}, u_{2}\right)\right) \leq C n\left[G\left(u_{2}\right)-G\left(u_{1}\right)\right] .
\end{aligned}
$$

First, note that the following two facts. One is that for $k \geq 1$

$$
P\left(r_{0}<y_{0} \leq r_{0}+u, r_{0}<y_{k} \leq r_{0}+u\right) \leq C u^{2}
$$

by a conditional argument. The other is that there exist two finite positive constants $m_{0}$ and $M_{0}$ such that

$$
m_{0}\left(u_{2}-u_{1}\right) \leq G\left(u_{2}\right)-G\left(u_{1}\right) \leq M_{0}\left(u_{2}-u_{1}\right)
$$

for $r_{0}-1 \leq u_{1} \leq u_{2} \leq r_{0}+1$, since the density of $y_{t}$ is continuous and positive on $R$ by Assumption 3.2 and the convolution property.

For (A.4), by Hölder's inequality and Assumptions 3.33.4 , it follows that

$$
E\left[A_{t}\left(u_{1}, u_{2}\right)\right] \leq C\left(u_{2}-u_{1}\right)^{3 / 2} \leq C\left[G\left(u_{2}\right)-G\left(u_{1}\right)\right] .
$$

The first inequality in (A.7) implies that it is not necessary to subtract $E\left[A_{t}\left(u_{1}, u_{2}\right)\right]$ from $A_{t}\left(u_{1}, u_{2}\right)$.

For (A.5), by Hölder's inequality and Assumptions 3.33.4 again, we have

$$
\begin{aligned}
\operatorname{Var}\left(A_{t}\left(u_{1}, u_{2}\right)\right) & \leq E\left[A_{t}^{2}\left(u_{1}, u_{2}\right)\right] \\
& \leq C\left(E\left\|\mathbf{Z}_{t}\right\|^{4}\right)^{1 / 2}\left[G\left(u_{2}\right)-G\left(u_{1}\right)\right] .
\end{aligned}
$$

For (A.6), by a simple calculation, we have

$$
\begin{aligned}
& \left|E\left(A_{t} \mid \mathcal{G}_{0}\right)-E\left(A_{t}\right)\right| \\
& \quad \leq C t \rho^{t}\left(1+\left\|\mathbf{Z}_{0}\right\|\right)+C \rho^{t}+C \rho^{t} \sum_{j=0}^{\infty} \rho^{j}\left\|\mathbf{Z}_{-j}\right\|,
\end{aligned}
$$

where $\mathcal{G}_{0}=\sigma\left(\mathbf{Z}_{t}, t \leq 0\right)$, which implies that

$$
\begin{aligned}
\left|\operatorname{Cov}\left(A_{0}\left(u_{1}, u_{2}\right), A_{t}\left(u_{1}, u_{2}\right)\right)\right| & \leq E\left\{A_{0}\left|E\left(A_{t} \mid \mathcal{G}_{0}\right)-E A_{t}\right|\right\} \\
& \leq C t \rho^{t}\left[G\left(u_{2}\right)-G\left(u_{1}\right)\right] .
\end{aligned}
$$

Thus,

$$
\begin{aligned}
\operatorname{Var} & \left(\sum_{t=1}^{n} A_{t}\left(u_{1}, u_{2}\right)\right) \\
= & n \operatorname{Var}\left(A_{t}\left(u_{1}, u_{2}\right)\right) \\
& +2 \sum_{k=1}^{n-1}(n-k) \operatorname{Cov}\left(A_{0}\left(u_{1}, u_{2}\right), A_{k}\left(u_{1}, u_{2}\right)\right) \\
\leq & C n\left\{\sum_{k=1}^{\infty} k \rho^{k}\right\}\left[G\left(u_{2}\right)-G\left(u_{1}\right)\right] \leq C n\left[G\left(u_{2}\right)-G\left(u_{1}\right)\right] .
\end{aligned}
$$

The proof of is complete.

Proof of Theorem 3.2 (i). Since $\widehat{\boldsymbol{\theta}}_{n}$ is consistent, we restrict $\Theta$ to an open neighborhood of $\boldsymbol{\theta}_{0}$, defined by $U_{\delta}=\{\boldsymbol{\theta} \in$ $\left.\Theta:\left\|\boldsymbol{\lambda}-\boldsymbol{\lambda}_{0}\right\|<\delta,\left|r-r_{0}\right|<\delta\right\}$ for some $0<\delta<1$ to be determined later. Then it suffices to show that there exist constants $B>0, \gamma>0$, such that for $n$ large enough

$$
P\left(\inf _{\substack{B / n<\left|r-r_{0}\right| \leq \delta \\ \boldsymbol{\theta} \in U_{\delta}}} \frac{L_{n}(\boldsymbol{\lambda}, r)-L_{n}\left(\boldsymbol{\lambda}, r_{0}\right)}{n G\left(\left|r-r_{0}\right|\right)}>\gamma\right)>1-4 \varepsilon .
$$

Here, we only treat the case $r>r_{0}$. The proof for the case $r<r_{0}$ is similar.

Let $r=r_{0}+u$ for some $0<u<1$. Decompose $L_{n}(\boldsymbol{\lambda}, r)-$ $L_{n}\left(\boldsymbol{\lambda}, r_{0}\right)$ as

$$
\begin{aligned}
L_{n}(\boldsymbol{\lambda}, r)-L_{n}\left(\boldsymbol{\lambda}, r_{0}\right) \\
=\left\{\left[L_{n}(\boldsymbol{\lambda}, r)-L_{n}\left(\boldsymbol{\lambda}, r_{0}\right)\right]-\left[L_{n}\left(\boldsymbol{\lambda}_{0}, r\right)-L_{n}\left(\boldsymbol{\lambda}_{0}, r_{0}\right)\right]\right\} \\
\quad+\left[L_{n}\left(\boldsymbol{\lambda}_{0}, r\right)-L_{n}\left(\boldsymbol{\lambda}_{0}, r_{0}\right)\right] \\
\equiv L_{n}^{(1)}(\boldsymbol{\lambda}, r)+L_{n}^{(2)}(r) .
\end{aligned}
$$

We first consider $L_{n}^{(2)}(r)$. Note that

$$
\begin{aligned}
\varepsilon_{t}\left(\boldsymbol{\lambda}_{0}, r\right)-\varepsilon_{t} & =f\left(\mathbf{Z}_{t-1}\right)+g\left(\mathbf{Z}_{t-1}\right)\left[\varepsilon_{t-1}\left(\boldsymbol{\lambda}_{0}, r\right)-\varepsilon_{t-1}\right] \\
& =\sum_{j=0}^{\infty}\left\{\prod_{i=1}^{j} g\left(\mathbf{Z}_{t-i}\right)\right\} f\left(\mathbf{Z}_{t-1-j}\right)
\end{aligned}
$$


where

$$
\begin{aligned}
f\left(\mathbf{Z}_{t-1}\right)= & \left\{\sum_{i=1}^{p}\left(\phi_{2 i 0}-\phi_{1 i 0}\right) y_{t-i}+\left(\psi_{20}-\psi_{10}\right) \varepsilon_{t-1}\right\} \\
& \times I\left(r_{0}<y_{t-d} \leq r\right), \\
g\left(\mathbf{Z}_{t-1}\right)= & -\left[\psi_{20}+\left(\psi_{10}-\psi_{20}\right) I\left(y_{t-d} \leq r\right)\right] .
\end{aligned}
$$

By Assumption 3.5, there exists $\beta>0$ such that $\left(\left(\phi_{10}-\right.\right.$ $\left.\left.\boldsymbol{\phi}_{20}\right)^{\prime},\left(\boldsymbol{\psi}_{10}-\boldsymbol{\psi}_{20}\right)^{\prime}\right)^{\prime} \mathbf{Z}$ is bounded away from 0 for all $\mathbf{Z}$ satisfying $\left\|\mathbf{Z}-\mathbf{Z}^{*}\right\| \leq \beta$ with $\mathbf{Z}^{*}=\left(\mathbf{w}^{\prime}, \mathbf{a}^{\prime}\right)^{\prime}$. Thus,

$$
\begin{aligned}
& \varepsilon_{t}^{2}\left(\boldsymbol{\lambda}_{0}, r\right)-\varepsilon_{t}^{2} \\
& \geq {\left[f\left(\mathbf{Z}_{t-1}\right)\right]^{2} I\left(\left\|\mathbf{Z}_{t-1}-\mathbf{Z}^{*}\right\| \leq \beta\right)+2 \varepsilon_{t}\left[\varepsilon_{t}\left(\boldsymbol{\lambda}_{0}, r\right)-\varepsilon_{t}\right] } \\
&+2 f\left(\mathbf{Z}_{t-1}\right) I\left(\left\|\mathbf{Z}_{t-1}-\mathbf{Z}^{*}\right\| \leq \beta\right) \\
& \times g\left(\mathbf{Z}_{t-1}\right)\left[\varepsilon_{t-1}\left(\boldsymbol{\lambda}_{0}, r\right)-\varepsilon_{t-1}\right] .
\end{aligned}
$$

Clearly, $\left|f\left(\mathbf{Z}_{t-1}\right)\right| \leq C\left\|\mathbf{Z}_{t-1}\right\| I\left(r_{0}<y_{t-d} \leq r\right)$ and $\left|g\left(\mathbf{Z}_{t-1}\right)\right| \leq \rho<1$ by the compactness of $\Theta$. Then

$$
\begin{aligned}
2 \mid f & \left(\mathbf{Z}_{t-1}\right) I\left(\left\|\mathbf{Z}_{t-1}-\mathbf{Z}^{*}\right\| \leq \beta\right) g\left(\mathbf{Z}_{t-1}\right)\left[\varepsilon_{t-1}\left(\boldsymbol{\lambda}_{0}, r\right)-\varepsilon_{t-1}\right] \mid \\
& \leq C I\left(r_{0}<y_{t-d} \leq r\right) \sum_{j=0}^{\infty} \rho^{j}\left\|\mathbf{Z}_{t-2-j}\right\| I\left(r_{0}<y_{t-1-d-j} \leq r\right) \\
& =C A_{t}(0, u)
\end{aligned}
$$

and there exists a positive constant $c_{0}$ such that

$$
\begin{aligned}
& {\left[f\left(\mathbf{Z}_{t-1}\right)\right]^{2} I\left(\left\|\mathbf{Z}_{t-1}-\mathbf{Z}^{*}\right\| \leq \beta\right)} \\
& \quad \geq c_{0} I\left(r_{0}<y_{t-d} \leq r,\left\|\mathbf{Z}_{t-1}-\mathbf{Z}^{*}\right\| \leq \beta\right) \\
& \quad=c_{0} I^{*}\left(r_{0}<y_{t-d} \leq r\right) .
\end{aligned}
$$

Hence,

$$
\begin{aligned}
\frac{L_{n}^{(2)}(r)}{n G(u)} \geq & \frac{c_{0} G^{*}(u)}{G(u)} \frac{\sum_{t=1}^{n} I^{*}\left(r_{0}<y_{t-d} \leq r_{0}+u\right)}{n G^{*}(u)} \\
& -C \frac{\sum_{t=1}^{n} A_{t}(0, u)}{n G(u)} \\
& +2 \frac{\sum_{t=1}^{n} \varepsilon_{t}\left[\varepsilon_{t}\left(\boldsymbol{\lambda}_{0}, r_{0}+u\right)-\varepsilon_{t}\right]}{n G(u)}
\end{aligned}
$$

Note that

$$
\lim _{u \downarrow 0} \frac{G^{*}(u)}{G(u)}=P\left(\left\|\mathbf{Z}_{t-1}-\mathbf{Z}^{*}\right\| \leq \beta \mid y_{t-d}=r_{0}^{+}\right)>0
$$

by a conditional argument for the inequality above. Write the limit as $a_{0}$. Then there exists a $\delta>0$ small enough such that $c_{0} G^{*}(u) / G(u) \geq c_{0} a_{0} / 2$ on $[0, \delta]$. By Lemma A.1, it follows that

$$
P\left(\inf _{B / n<u \leq \delta} \frac{L_{n}^{(2)}(r)}{n G(u)}>\frac{c_{0} a_{0}}{4}\right)>1-3 \varepsilon .
$$

Next, we consider $L_{n}^{(1)}(\boldsymbol{\lambda}, r)$. Clearly,

$$
\begin{aligned}
\frac{1}{n} & L_{n}^{(1)}(\boldsymbol{\lambda}, r) \\
& =\frac{1}{n} \sum_{t=1}^{n} \int_{0}^{1}\left[\frac{\partial \varepsilon_{t}^{2}\left(\boldsymbol{\lambda}_{v}, r\right)}{\partial \boldsymbol{\lambda}^{\prime}}-\frac{\partial \varepsilon_{t}^{2}\left(\boldsymbol{\lambda}_{v}, r_{0}\right)}{\partial \boldsymbol{\lambda}^{\prime}}\right]\left(\boldsymbol{\lambda}-\boldsymbol{\lambda}_{0}\right) d v
\end{aligned}
$$

where $\boldsymbol{\lambda}_{v}=\boldsymbol{\lambda}_{0}+v\left(\boldsymbol{\lambda}-\boldsymbol{\lambda}_{0}\right)$. Similarly, we can prove that

$$
\sup _{\substack{B / n<u \leq \delta \\ \boldsymbol{\theta} \in U_{\delta}}} \frac{\left|L_{n}^{(1)}(\boldsymbol{\lambda}, r)\right|}{n G(u)}=O_{p}\left(\sup _{\boldsymbol{\theta} \in U_{\delta}}\left\|\boldsymbol{\lambda}-\boldsymbol{\lambda}_{0}\right\|\right)=O_{p}(\delta) .
$$

Clearly,

$$
\begin{aligned}
\inf _{\substack{B / n<u \leq \delta \\
\boldsymbol{\theta} \in U_{\delta}}} \frac{L_{n}(\boldsymbol{\lambda}, r)-L_{n}\left(\boldsymbol{\lambda}, r_{0}\right)}{n G(u)} \\
\quad \geq \inf _{B / n<u \leq \delta} \frac{L_{n}^{(2)}(r)}{n G(u)}-\sup _{\substack{B / n<u \leq \delta \\
\boldsymbol{\theta} \in U_{\delta}}} \frac{\left|L_{n}^{(1)}(\boldsymbol{\lambda}, r)\right|}{n G(u)} .
\end{aligned}
$$

Let $\gamma=c_{0} a_{0} / 8>0$. Then, for sufficiently small $\delta>0$, (A.8) holds. The proof of (i) is complete.

For the proof of Theorem 3.2 (ii), it is similar to that of Theorem 2.2 (ii) in Li, Ling and $\mathrm{Li}$ (2010). Hence it is omitted.

\section{APPENDIX B. PROOF OF THEOREM 3.3}

Here, we give an outline of the proof, which is similar to that of Theorem $2.3 \mathrm{in} \mathrm{Li}$, Ling and $\mathrm{Li}$ (2010). First of all, we can prove that $d\left(\widetilde{L}_{n}(z), \wp_{n}(z)\right) \rightarrow 0$ in probability, where $d(\cdot, \cdot)$ is the Skorokhod metric in $D(R)$. Secondly, we show that $\wp_{n}(z)$ converges weakly to a two-sided compound Poisson process $\wp(z)$ in $D(R)$. The tightness of $\left\{\wp_{n}(z)\right\}$ can be verified by Theorem 5 in Kushner (1984, pp. 32). To characterize convergence of finite dimensional distributions of $\left\{\wp_{n}(z)\right\}$, we study the truncated process $\left\{\wp_{n}^{(a)}(z)\right\}$ for $a>0$ large enough, defined similarly to $\left\{\wp_{n}(z)\right\}$ with the jumps being replaced by $\zeta_{1 t}^{(a)} \equiv \zeta_{1 t} I\left(\left|\zeta_{1 t}\right| \leq a\right)$. Now we consider the weak convergence of $\left\{\wp_{n}^{(a)}(z)\right\}$ as $n \rightarrow \infty$ for each $a>0$. This takes two steps. (i). Proving the tightness of $\left\{\wp_{n}^{(a)}(z)\right\}$. This can be done by Theorem 5 in Kushner (1984, pp. 32) again; (ii). Characterizing the convergence of finite dimensional distributions of $\left\{\wp_{n}^{(a)}(z)\right\}$, which can be completed by verifying Assumptions A.1-A.4 in the Appendix in Li, Ling and $\mathrm{Li} \mathrm{(2010).} \mathrm{Thus} \mathrm{we} \mathrm{have} \mathrm{shown} \mathrm{that}$ $\left\{\wp_{n}^{(a)}(z)\right\}$ converges weakly to a two-sided compound Poisson process $\wp^{(a)}(z)$, having the jump rate $\kappa_{1}^{(a)} \pi\left(r_{0}\right)$ and the jump distribution $Q_{1}^{(a)}$ on the left side (i.e., $z<0$ ) and the jump rate $\kappa_{2}^{(a)} \pi\left(r_{0}\right)$ and the jump distribution $Q_{2}^{(a)}$ on the right side (i.e., $z>0$ ), where $\kappa_{j}^{(a)}=P\left(\left|\zeta_{j t}\right| \leq a \mid y_{t-d}=r_{0}\right)$ and $Q_{j}^{(a)}(A)=P\left(\zeta_{j t} \in A\left|y_{t-d}=r_{0},\right| \zeta_{j t} \mid \leq a\right)$ for any Borel set $A$ and $j=1,2$. Note that $\kappa_{j}^{(a)} \rightarrow 1$ and $Q_{j}^{(a)} \rightarrow F_{j}\left(\cdot \mid r_{0}\right)$ 
as $a \rightarrow \infty$, we have that $\wp^{(a)}(z)$ converges weakly to $\wp(z)$ in (3.5) by Theorem 16 in Pollard (1984, pp. 134). In addition, $\lim _{a \rightarrow \infty} \limsup _{n \rightarrow \infty} P\left(d\left(\wp_{n}^{(a)}(z), \wp_{n}(z)\right)>\epsilon\right)=0$ for any $\epsilon>0$. Hence, $\wp_{n}(z)$ converges weakly to $\wp(z)$ by Theorem 3.2 in Billingsley (1999, pp. 28). The remainder is the same as Theorem 2 in Chan (1993).

\section{ACKNOWLEDGEMENTS}

W.K. Li would like to thank Hong Kong Research Grants Commission Grant HKU7036/06P for partial support. S. Ling thanks Hong Kong Research Grants Commission Grants HKUST601607 and HKUST602609 for partial support. The authors also thank two anonymous referees and the Editor for their helpful comments that improved the presentation.

\section{Received 28 October 2010}

\section{REFERENCES}

AN, H. Z. and HuAng, F. C. (1996). The geometrical ergodicity of nonlinear autoregressive models. Statist. Sinica 6 943-956. MR1422412

Billingsley, P. (1999). Convergence of Probability Measures, 2nd ed. John Wiley \& Sons, New York. MR1700749

Brockwell, P. J., Liu, J. and Tweedie, R. L. (1992). On the existence of stationary threshold autoregressive moving-average processes. J. Time Ser. Anal. 13 95-107. MR1165659

Chan, K. S. (1989). A note on the geometric ergodicity of a Markov chain. Adv. in Appl. Probab. 21 702-704. MR1013658

Chan, K. S. (1993). Consistency and limiting distribution of the least squares estimator of a threshold autoregressive model. Ann. Statist. 21 520-533. MR1212191

Chan, K. S., Petruccelli, J. D., Tong, H. and Woolford, S. W. (1985). A multiple-threshold AR(1) model. J. Appl. Probab. 22 267279. MR0789351

Chan, K. S. and Tong, H. (1985). On the use of the deterministic Lyapunov function for the ergodicity of stochastic difference equations. Adv. in Appl. Probab. 17 666-678. MR0798881

Chan, K. S. and Tong, H. (2010). A note on the invertibility of nonlinear ARMA models. J. Statist. Plann. Inference 140 3709-3714. MR2674159

Chan, K. S. and Tsay, R. S. (1998). Limiting properties of the least squares estimator of a continuous threshold autoregressive model. Biometrika 85 413-426. MR1649122

Chen, R. and Tsay, R. S. (1991). On the ergodicity of TAR(1) processes. Ann. Appl. Probab. 1 613-634. MR1129777

Cont, R. and Tankov, P. (2004). Financial Modelling with Jump Processes. Chapman \& Hall. MR2042661

FAn, J. and YAO, Q. (2003). Nonlinear Time Series: Nonparametric and Parametric Methods. Springer-Verlag, New York. MR1964455

Ghaddar, D. K. and Tong, H. (1981). Data transformation and selfexciting threshold autoregression. J. R. Stat. Soc. Ser. C: Appl. Statist. $30238-248$.

Giovanni, F. (2005). On the stability of nonlinear ARMA models. Technical Report.

GooiJer, D. J. G. (1998). On threshold moving-average models. J. Time Ser. Anal. 19 1-18. MR1624163

Kushner, H. J. (1984). Approximation and Weak Convergence Methods for Random Processes, with Applications to Stochastic Systems Theory. MIT. MR0741469

Li, D. and Ling, S. (2010). Inference for multiple-regime threshold autoregressive models. Working Paper in HKUST.
LI, D., Ling, S. and LI, W. K. (2010). Asymptotic theory on the least squares estimation of threshold moving-average models. Working Paper in HKUST.

Li, D., Ling, S. and Tong, H. (2011). On moving-average models with feedback. To appear in Bernoulli. MR2307400

LI, G. D. and LI, W. K. (2008). Testing for threshold moving average with conditional heteroscedasticity. Statist. Sinica 18 647-665. MR2432284

LI, G. D. and LI, W. K. (2011). Testing for linear time series models against its threshold extension. Biometrika 98 243-250.

LING, S. (1999). On the probabilistic properties of a double threshold ARMA conditional heteroskedastic model. J. Appl. Probab. 36 688705. MR1737046

Ling, S. and Tong, H. (2005). Testing for a linear MA model against threshold MA models. Ann. Statist. 33 2529-2552. MR2253094

Ling, S., Tong, H. and LI, D. (2007). Ergodicity and invertibility of threshold moving-average models. Bernoulli 13 161-168. MR2307400

LiU, J. and Susko, E. (1992). On strict stationarity and ergodicity of a nonlinear ARMA model. J. Appl. Probab. 29 363-373. MR1165221

Meyn, S. P. and Tweedie, R. L. (1993). Markov Chains and Stochastic Stability. Springer-Verlag. MR1287609

Petruccelli, J. D. (1986). On the consistency of least squares estimators for a threshold AR(1) model. J. Time Ser. Anal. 7 269-278. MR0883010

Pollard, D. (1984). Convergence of Stochastic Processes. SpringerVerlag. MR0762984

QIAN, L. (1998). On maximum likelihood estimators for a threshold autoregression. J. Statist. Plann. Inference 75 21-46. MR1671666

Seijo, E. and SEn, B. (2011). A continuous mapping theorem for the smallest argmax functional. Electron. J. Stat. 5 421-439.

Silverman, B. (1978). Weak and strong uniform consistency of the kernel estimate of a density and its derivatives. Ann. Statist. 6 177184. MR0471166

Tong, H. (1978). On a threshold model. In Pattern Recognition and Signal Processing. (Chen, C. H. ed.). Sijthoff and Noordhoff, Amsterdam, pp. 575-586. MR0552641

Tong, H. (1990). Non-linear Time Series: A Dynamical System Approach. Oxford University Press, New York. MR1079320

Tong, H. (2011). Threshold models in time series analysis - 30 years on. To appear in Statistics and its Interface.

TSAY, R. S. (1989). Testing and modeling threshold autoregressive processes.J. Amer. Statist. Assoc. 84 231-240. MR0999683

\section{Dong Li}

Department of Mathematics

Hong Kong University of Science and Technology

Hong Kong

E-mail address: li20072010@gmail.com

Wai Keung Li

Department of Statistics and Actuarial Science

The University of Hong Kong

Pokfulam Road

Hong Kong

E-mail address: hrntlwk@hku.hk

url: http://www3.hku.hk/statisti/staff/hrntlwk/ index.php

Shiqing Ling

Department of Mathematics

Hong Kong University of Science and Technology

Hong Kong

E-mail address: maling@ust.hk

url: http://www.math.ust.hk/ maling/ 